\title{
Adjuvanting a subunit COVID-19 vaccine to induce protective immunity
}

https://doi.org/10.1038/s41586-021-03530-2

Received: 10 February 2021

Accepted: 9 April 2021

Published online: 19 April 2021

Check for updates

\author{
Prabhu S. Arunachalam ${ }^{1}$, Alexandra C. Walls ${ }^{2}$, Nadia Golden $^{3}$, Caroline Atyeo $^{4}$, \\ Stephanie Fischinger ${ }^{4}$, Chunfeng Li ${ }^{1}$, Pyone Aye ${ }^{3}$, Mary Jane Navarro ${ }^{2}$, Lilin Lai ${ }^{5}$, \\ Venkata Viswanadh Edara ${ }^{5}$, Katharina Röltgen ${ }^{6}$, Kenneth Rogers ${ }^{7}$, Lisa Shirreff ${ }^{7}$, \\ Douglas E. Ferrell', Samuel Wrenn ${ }^{2,8}$, Deleah Pettie ${ }^{2,8}$, John C. $\mathrm{Kraft}^{2,8}$, \\ Marcos C. Miranda ${ }^{2,8}$, Elizabeth Kepl ${ }^{2,8}$, Claire Sydeman ${ }^{2,8}$, Natalie Brunette ${ }^{2,8}$, \\ Michael Murphy ${ }^{2,8}$, Brooke Fiala $^{2,8}$, Lauren Carter ${ }^{2,8}$, Alexander G. White ${ }^{9,10}$, Meera Trisal', \\ Ching-Lin Hsieh" ${ }^{11}$, Kasi Russell-Lodrigue ${ }^{3}$, Christopher Monjure ${ }^{3}$, Jason Dufour ${ }^{3}$, \\ Skye Spencer ${ }^{3}$, Lara Doyle-Meyers ${ }^{3}$, Rudolph P. Bohm ${ }^{3}$, Nicholas J. Maness ${ }^{3}$, Chad Roy ${ }^{3}$, \\ Jessica A. Plante ${ }^{12}$, Kenneth S. Plante ${ }^{12}$, Alex Zhu ${ }^{4}$, Matthew J. Gorman ${ }^{4}$, Sally Shin ${ }^{4}$, \\ Xiaoying Shen ${ }^{13}$, Jane Fontenot ${ }^{7}$, Shakti Gupta ${ }^{14}$, Derek T. O'Hagan ${ }^{15}$, Robbert Van Der Most ${ }^{16}$, \\ Rino Rappuoli ${ }^{17}$, Robert L. Coffman ${ }^{18}$, David Novack ${ }^{18}$, Jason S. McLellan ${ }^{11}$, \\ Shankar Subramaniam ${ }^{14}$, David Montefiori ${ }^{13}$, Scott D. Boyd ${ }^{6}$, JoAnne L. Flynn ${ }^{9}$, \\ Galit Alter ${ }^{4}$, Francois Villinger ${ }^{7}$, Harry Kleanthous ${ }^{19}$, Jay Rappaport ${ }^{3,20}$, Mehul S. Suthar ${ }^{5}$, \\ Neil P. King ${ }^{2,7}$, David Veesler ${ }^{2}$ \& Bali Pulendran ${ }^{1,6,21 \bowtie}$
}

The development of a portfolio of COVID-19 vaccines to vaccinate the global population remains an urgent public health imperative ${ }^{1}$. Here we demonstrate the capacity of a subunit vaccine, comprising the SARS-CoV-2 spike protein receptor-binding domain displayed on an 153-50 protein nanoparticle scaffold (hereafter designated RBD-NP), to stimulate robust and durable neutralizing-antibody responses and protection against SARS-CoV-2 in rhesus macaques. We evaluated five adjuvants including Essai O/W 1849101, a squalene-in-water emulsion; ASO3, an $\alpha$-tocopherol-containing oil-in-water emulsion; AS37, a Toll-like receptor 7 (TLR7) agonist adsorbed to alum; CpG1018-alum, a TLR9 agonist formulated in alum; and alum. RBD-NP immunization with ASO3, CpG1018-alum, AS37 or alum induced substantial neutralizing-antibody and CD4 $\mathrm{T}$ cell responses, and conferred protection against SARS-CoV- 2 infection in the pharynges, nares and bronchoalveolar lavage. The neutralizing-antibody response to live virus was maintained up to 180 days after vaccination with RBD-NP in AS03 (RBDNP-AS03), and correlated with protection from infection. RBD-NP immunization cross-neutralized the B.1.1.7 SARS-CoV-2 variant efficiently but showed a reduced response against the B.1.351 variant. RBD-NP-AS03 produced a 4.5 -fold reduction in neutralization of B.1.351 whereas the group immunized with RBD-NP-AS37 produced a 16-fold reduction in neutralization of B.1.351, suggesting differences in the breadth of the neutralizing-antibody response induced by these adjuvants. Furthermore, RBD-NP-ASO3 was as immunogenic as a prefusion-stabilized spike immunogen (HexaPro) with AS03 adjuvant. These data highlight the efficacy of the adjuvanted RBD-NP vaccine in promoting protective immunity against SARS-CoV-2 and have led to phase I/II clinical trials of this vaccine (NCT04742738 and NCT04750343).
Subunit vaccines are among the safest and most widely used vaccines, and have been highly effective against a variety of infectious diseases, such as hepatitis B, diphtheria, pertussis, tetanus and shingles in diverse age groups ${ }^{2}$. Therefore, the development of a safe and effective subunit vaccine against SARS-CoV-2 would represent an important step in controlling the COVID-19 pandemic. An essential component of subunit vaccines is the adjuvant, an immune-stimulatory agent that enhances the magnitude, quality and durability of the immune responses induced by vaccination, even with lower doses of antigen ${ }^{3}$. Alum, the most widely used adjuvant, has been used in billions of vaccine doses over the last century. During the past two decades, novel adjuvants have been developed including the $\alpha$-tocopherol-containing squalene-based oil-in-water adjuvant ASO3 and the TLR9 ligand CpG1018, which are included in licensed vaccines against pandemic 

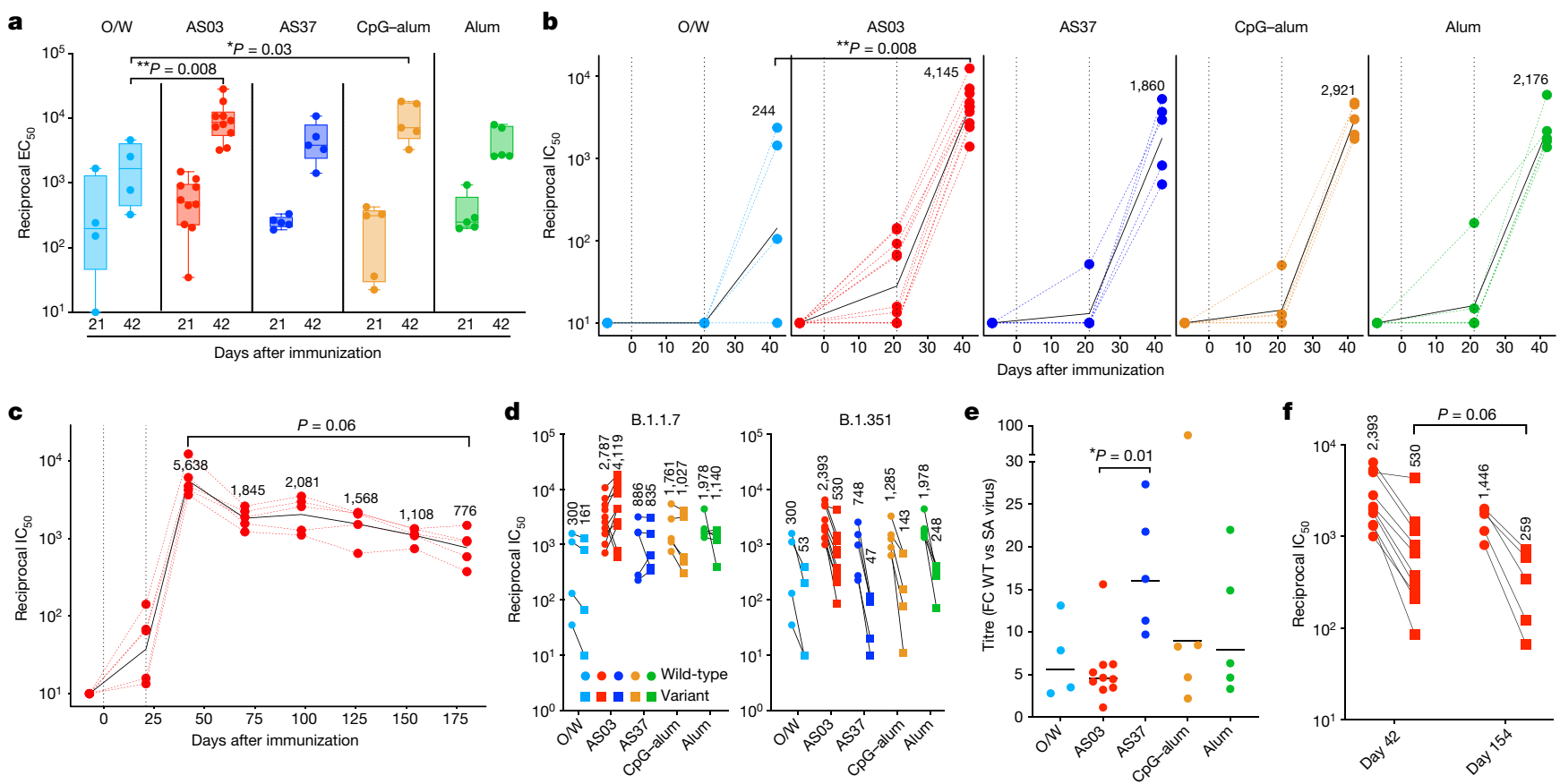

Fig. $1 \mid$ RBD-NP immunization induces robust antibody responses. a, SARS-CoV-2 spike-specific IgG titres in sera following immunization with RBD-NP and the indicated adjuvant measured by ELISA. Boxes represent median, and 25 th and 75 th percentiles and whiskers show the range. b, c, Serum neutralizing-antibody titres determined by authentic SARS-CoV-2 assay at indicated time points. The black line represents the geometric mean of all data points and numbers show GMT on day 42 (b) and subsequent time points (c). $\mathrm{IC}_{50}$, half-maximal inhibitory concentration. d, Neutralizing-antibody titres against live wild-type (circles) or B.1.1.7 or B.1.351 variant (squares) SARS-CoV-2 virus measured in serum on day 42 . Numbers indicate GMT.e, The fold change in neutralizing-antibody titres for the B.1.351 variant versus wild-type SARS-CoV-2.f, Serum neutralizing-antibody titres against the live wild-type (circles) or B.1.351 variant (squares) viruses measured on day 42 or day 154. a, b, e, Two-sided Mann-Whitney rank-sum test. c,f, Two-sided Wilcoxon matched-pairs signed-rank test. Each symbol represents one monkey. $n=4$ (O/W), 10 (AS03) and 5 (all other groups). influenza and hepatitis B, respectively. In particular, AS03 and CpG1018 are currently being developed as adjuvants for use in candidate subunit SARS-CoV-2 vaccines ${ }^{3,4}$; however, their capacity to stimulate protective immunity against SARS-CoV-2 remains unknown. In this Article, we evaluate the use of AS03, CpG1018 formulated in alum (CpG-alum), squalene-in-water emulsion (O/W), AS37 and alum as adjuvants for a subunit vaccine in which 60 copies of the SARS-CoV-2 RBD are displayed in a highly immunogenic array using a computationally designed self-assembling protein nanoparticle (RBD-NP) ${ }^{5}$, and demonstrate protective immunity against SARS-CoV-2 in non-human primates.

\section{Robust and durable neutralizing-antibody responses}

To assess the immunogenicity and protective efficacy of RBD-NP vaccination with different adjuvants, we immunized 29 rhesus macaques with $25 \mu \mathrm{g}$ RBD antigen (71 $\mu$ g total RBD-NP immunogen) (Extended Data Fig. 1a-d) formulated with one of the five adjuvants: O/W, ASO3, AS37, CpG-alum or alum (Extended Data Fig. 1e). Four additional monkeys were administered saline as a control (Extended Data Table 1). All immunizations were administered via the intramuscular route in forelimbs on days 0 and 21. Four weeks after the booster immunization, the monkeys were challenged with SARS-CoV-2 via the intratracheal-intranasal routes. Five of the ten monkeys immunized with RBD-NP-AS03 were not challenged, to enable longitudinal analysis of the durability of the immune responses.

Evaluation of binding-antibody responses showed that spike-specific IgG was detected 21 days after primary immunization in all vaccination groups and the response increased in magnitude after boosting (Fig. 1a). RBD-NP-ASO3 induced the highest magnitude (geometric mean titre $(\mathrm{GMT})$ half-maximal effective concentration $\left(\mathrm{EC}_{50}\right) 1: 8,551$ ) and RBD-NP-O/W induced the lowest $\left(\mathrm{GMT} \mathrm{EC}_{50} 1: 1,308\right)$ on day 42. The 153-50 nanoparticle scaffold also elicited an antibody response in all groups, which correlated with spike-specific antibody responses (Extended Data Fig. 1f, g). Immunization with RBD-NP induced detectable neutralizing-antibody responses against a SARS-CoV-2 spike pseudotyped virus ${ }^{6}$ in most of the monkeys (except in the RBD-NP-O/W group after primary immunization), which significantly increased in all groups after the booster immunization (Extended Data Fig. 2a). The neutralizing-antibody titres in all groups except the RBD-NP-O/W group were higher than that of 4 samples from convalescent human individuals who had suffered mild-to-moderate COVID-19 (GMT 1:76) and NIBSC control human convalescent plasma (NIBSC code 20/130, neutralizing-antibody titre 1:241) (Extended Data Fig. 2b) assayed simultaneously. Next, we measured neutralizing-antibody responses against authentic SARS-CoV-2 virus using focus reduction neutralization titre (FRNT) assay ${ }^{7}$, which was used to analyse the recent clinical trials of the Moderna mRNASARS-CoV-2 vaccine ${ }^{8,9}$. Consistent with the pseudovirus neutralizing-antibody titres, vaccinations with all adjuvants induced robust neutralizing-antibody titres against live virus after the secondary immunization, with the RBD-NP-AS03 group showing the highest titres (GMT 1:4,145, Fig. 1b). There was a strong correlation between pseudovirus and live-virus neutralizing-antibody titres, as observed in previous studies ${ }^{10,11}$ (Extended Data Fig. 2c). In addition, there was a robust induction of RBD-NP-specific plasmablast responses four days after secondary immunization (Extended Data Fig. 2d), the magnitude of which correlated with the observed antibody responses (Extended Data Fig. 2e).

To determine the durability of the neutralizing-antibody responses, we monitored 5 monkeys immunized with RBD-NP-AS03 without challenge for 6 months. The pseudovirus neutralizing-antibody titres declined moderately until day 126 , but did not differ significantly between days 42 and 126 (Extended Data Fig. 2f). Neutralizing-antibody response measured against the authentic SARS-CoV-2 virus was durably maintained up to day 180 (Fig. 1c). The GMT titres decreased by 

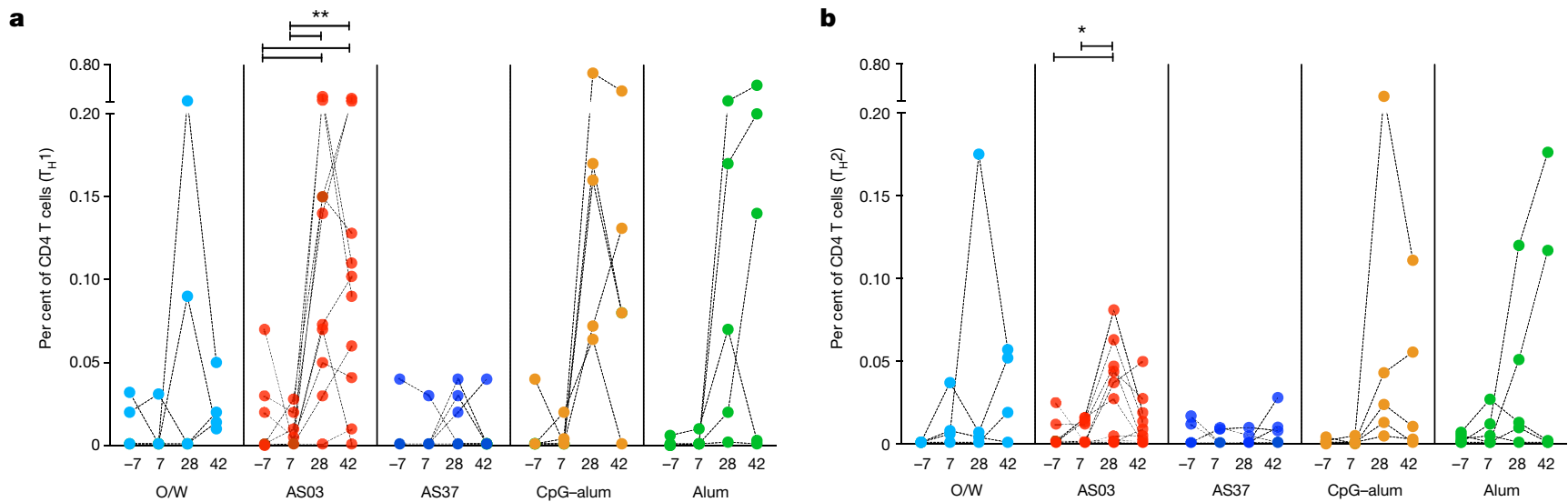

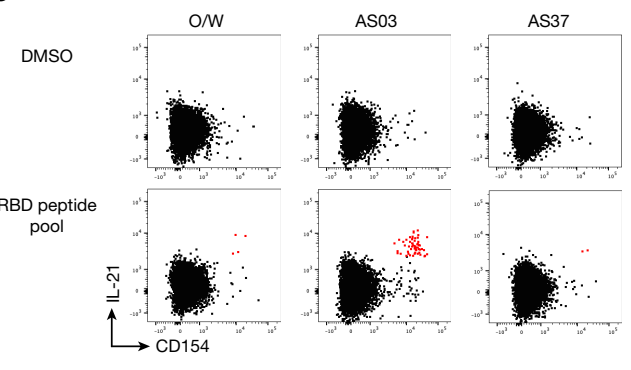

Fig. 2 | Cell-mediated immune responses to RBD-NP immunization. a, b, RBD-specific CD4 $\mathrm{T}$ cell responses measured in blood at indicated time points. CD4 T cells secreting IL-2, IFN $\gamma$, or TNF are plotted as $\mathrm{T}_{\mathrm{H}} 1$-type responses (a) and IL-4-producing CD4 T cells are shown as $\mathrm{T}_{\mathrm{H}} 2$-type responses (b).c, Flow cytometry plots showing expression of IL-21 and CD154 after ex vivo stimulation with DMSO (no peptide, top) or an overlapping peptide pool

7.2-fold between days 42 and 180. Furthermore, we observed little to no reduction in the efficiency of blocking of ACE2 binding to the RBD-a correlate of neutralizing-antibody response $\mathrm{e}^{12}$-by sera collected at these time points (Extended Data Fig. 2g). These results demonstrate that the RBD-NP-ASO3 immunization induces potent and durable neutralizing-antibody responses.

\section{Neutralization of variants of concern}

SARS-CoV-2 variants of concern, B.1.1.7 and B.1.351, first identified in the United Kingdom and South Africa, respectively, have since been found to be circulating globally. We used live virus and pseudovirus neutralization assays to evaluate sera from the immunized monkeys against these variants. Neutralizing-antibody titres against the B.1.1.7 variant were similar to those against the wild-type virus (Fig. 1d, left, Extended Data Fig. 2h); by contrast, titres against the B.1.351 variant were considerably lower (Fig. 1d, right, Extended Data Table 2), as seen in vaccinated humans ${ }^{13,14}$. Of note, there was a greater reduction of titre in the RBD-NP-AS37 group (median of 16-fold) compared with RBD-NP-ASO3 (4.5-fold) and other adjuvant groups (Fig. 1e). These data suggest that the adjuvants not only enhance immunogenicity, but also that different adjuvants vary in their potential to elicit neutralizing antibodies that provide a greater breadth of neutralization. Furthermore, the neutralizing-antibody response against the B.1.351 variant was as durable as that of the responses to the wild-type virus in the RBD-NP-ASO3 durability group (Fig. 1e).

\section{Induction of CD4 $T$ cell responses}

RBD-NP immunization resulted in an antigen-specific CD4 $\mathrm{T}$ cell response but limited CD8 $\mathrm{T}$ cell response following ex vivo stimulation d

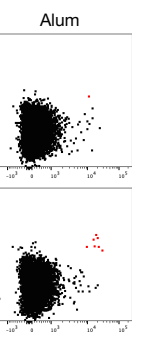

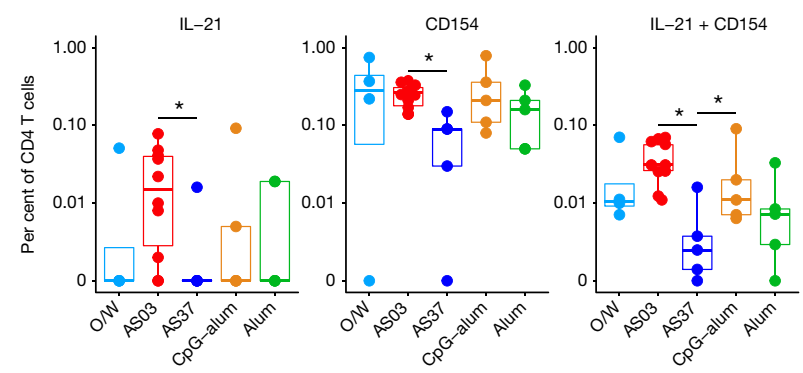

spanning the SARS-CoV-2 RBD (bottom). d, RBD-specific CD $154^{+}$and $\mathrm{CD} 154^{+} \mathrm{IL}_{-2} \mathrm{1}^{+} \mathrm{CD} 4 \mathrm{~T}$ cell responses measured in blood at day 28 after inoculation. Boxes show median, 25th and 75th percentiles and whiskers show the range. a, b, Two-sided Wilcoxon matched-pairs signed-rank test. d, Two-sided Mann-Whitney rank-sum test. ${ }^{*} P<0.05,{ }^{* *} P<0.01$. Each symbol represents one monkey. $n=4(\mathrm{O} / \mathrm{W}), 10$ (AS03) and 5 (all other groups). of peripheral blood mononuclear cells (PBMCs) with an overlapping peptide pool. RBD-specific CD4 responses were significantly enhanced only after the secondary immunization and were highest in the RBDNP-AS03 and RBD-NP-CpG-alum groups (Fig. 2a, b). The responses were dominated by IL-2- and TNF-secreting cells (Extended Data Fig. 3a), which remained detectable at day 42 ( 3 weeks after secondary immunization). There was a low but detectable IL-4 response in both the RBD-NP-ASO3 and RBD-NP-CpG-alum groups that peaked on day 28 but declined to near-baseline levels by day 42 (Fig. $2 \mathrm{~b}$ ). Whereas $75 \%$ of monkeys in the RBD-NP-alum group and $50 \%$ of those in the RBD-NP-O/W group showed induction of RBD-specific CD4 T cells, RBD-NP-AS37 induced a weak T cell response, less than that of the alum group, despite inducing potent antibody responses in all of the monkeys. This is in contrast to findings in mice with the same antigen ( $L$. Grigoryan et al., manuscript in preparation) and in non-human primates with an HIV antigen ${ }^{15}$. However, a direct comparison with the alum group is confounded by the larger amount of aluminium hydroxide in the alum group compared with the RBD-NP-AS37 group (Extended Data Fig.1e). We assessed the polyfunctional profile of antigen-specific CD4 T cells expressing IL-2, IFN $\gamma$, IL-4 and TNF (Extended Data Fig. 3b). Although $\mathrm{IL}^{+}{ }^{+}, \mathrm{TNF}^{+}$and IL-2 ${ }^{+} \mathrm{TNF}^{+}$cells formed the majority (around $70 \%$ ) in all adjuvant groups, differences between the groups were apparent. RBD-NP-AS03 elicited similar proportions of T helper $1\left(\mathrm{~T}_{\mathrm{H}} 1\right)$ and $\mathrm{T}_{\mathrm{H}} 2$ CD4 T cells, a balanced $\mathrm{T}_{\mathrm{H}} 1-\mathrm{T}_{\mathrm{H}} 2$ profile. $\mathrm{RBD}-\mathrm{NP}-\mathrm{CpG}$-alum showed a slightly higher $\mathrm{T}_{\mathrm{H}} 1$-type response, whereas RBD-NP-alum showed a higher $\mathrm{T}_{\mathrm{H}}$ 2-type response. We further extended our analyses to measure IL-21 and CD154, markers of circulating T follicular helper $\left(\mathrm{T}_{\mathrm{FH}}\right)$-like cells for their critical role in germinal centre formation and generation of durable $B$ cell responses. We observed detectable IL-21 responses in the RBD-NP-ASO3 and RBD-NP-CpG-alum groups (Fig. 2c). All cells secreting IL-21 were $\mathrm{CD} 154^{+}$, and the IL- $21^{+} \mathrm{CD} 154^{+}$double-positive cells 

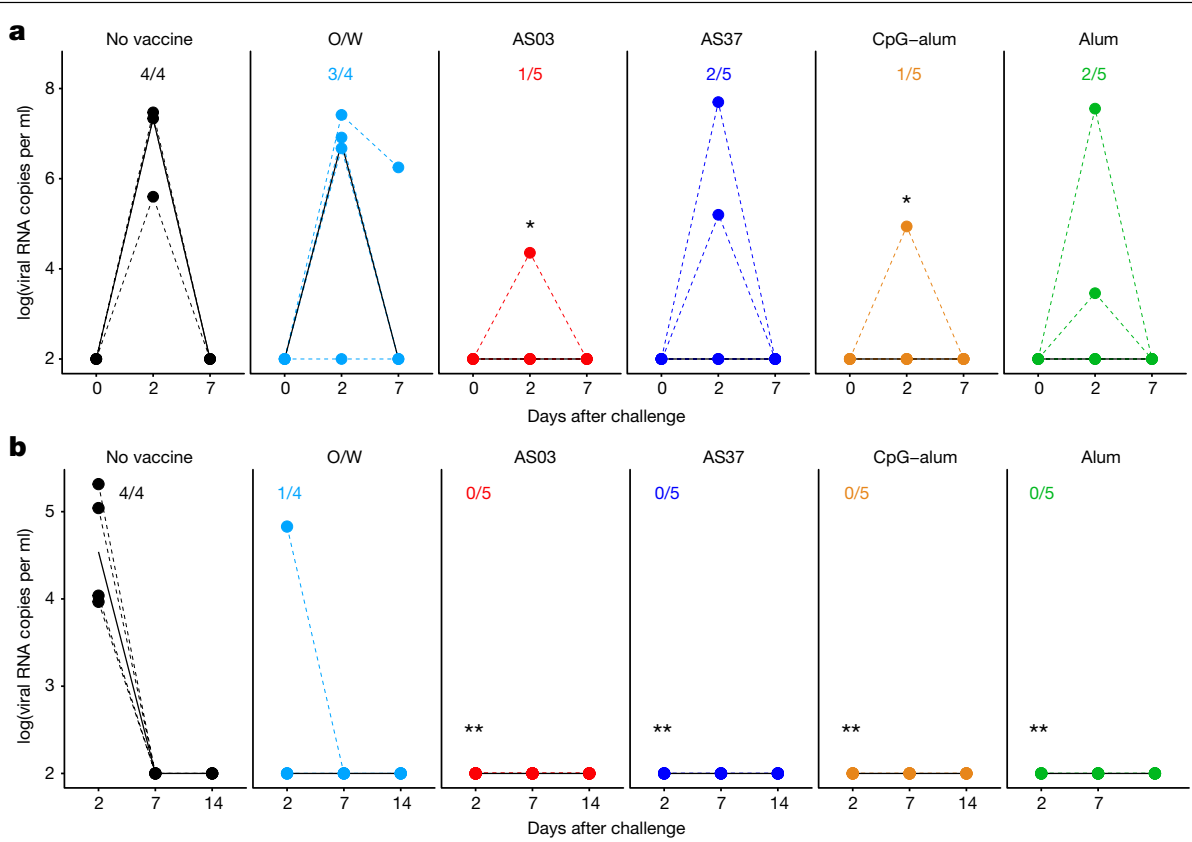

Fig. 3 | Protection against SARS-CoV-2 challenge. a, b, SARS-CoV-2 viral load in nares (a) and BAL fluid (b) measured using subgenomic PCR. The numbers shown on the plots denote number of infected monkeys/total number of monkeys within each group. Statistically significant differences compared with the no-vaccine control group by two-sided Mann-Whitney rank-sum test. ${ }^{*} P=0.01,{ }^{* *} P=0.008$. Each symbol represents a monkey. $n=4$ for control and $\mathrm{O} / \mathrm{W}, 5$ for all other groups. were significantly higher in the RBD-NP-ASO3 and RBD-NP-CpG-alum groups compared with the RBD-NP-AS37 group (Fig. 2d).

We also stimulated PBMCs with a peptide pool spanning the nanoparticle component sequences I53-50A and I53-50B to determine whether RBD-NP immunization induces T cells targeting the nanoparticle scaffold. We observed a significant proportion of CD4 T cells targeting the $153-50$ subunits with a response pattern similar to that of the RBD-specific T cells (Extended Data Fig. 3c). The frequencies of nanoparticle-specific CD4 T cells were about threefold higher than those of RBD-specific CD4 T cells (Extended Data Fig. 3d), an observation consistent with the RBD making up approximately one-third of the total peptidic mass of the immunogen.

\section{Protection against SARS-CoV-2 challenge}

To assess the protective efficacy, we challenged the monkeys four weeks after secondary immunization with SARS-CoV-2 via the intratracheal and intranasal routes. Two days after challenge, 4 out of 4 control monkeys had a subgenomic viral load $\left(3.1 \times 10^{5}\right.$ to $3.5 \times 10^{8}$ copies of the virus) in the pharyngeal and the nasal compartments. By day 7 , the viral load had decreased to baseline levels, consistent with previous studies ${ }^{16,17}$. All adjuvanted groups, except RBD-NP-O/W, afforded protection from infection (Fig. 3a, Extended Data Fig. 4a). None of the five monkeys in the RBD-NP-AS03 group had detectable viral RNA in pharyngeal swabs at any time and one monkey had a viral load in nasal swabs, at a level approximately 1,000 -fold lower than the median in control monkeys $\left(2.2 \times 10^{4}\right.$ versus $2.5 \times 10^{7}$ viral copies $)$. By contrast, viral RNA was detectable in pharyngeal and nasal swabs of all monkeys in the RBD$\mathrm{NP}-\mathrm{O} / \mathrm{W}$ group, albeit at lower levels than the control group. Only one out of five monkeys in the RBD-NP-CpG-alum group had detectable viral RNA in pharyngeal or nasal swabs. The RBD-NP-AS37 group and the RBD-NP-alum group also showed undetectable viral RNA in both compartments in 3 of the 5 monkeys. We measured the subgenomic viral RNA in bronchoalveolar lavage (BAL) fluid to assess protection in the lung. We used a more sensitive PCR assay to measure the product of the $N$ gene $^{18}$, as we found only two control monkeys with a positive viral load in the BAL fluid using E subgenomic RNA (sgRNA). Two days after the challenge, all 4 of the 4 control monkeys showed a viral load in the range of $10^{4}-10^{6}$ viral copies. By contrast, none of the monkeys in the vaccinated groups (except one in the RBD-NP-O/W group) showed any detectable virus, suggesting effective protection in the lower respiratory tracts of all vaccinated groups, including the RBD-NP-O/W group. There were no signs of clinical disease in any monkeys, whether or not they had been vaccinated (Extended Data Fig. 4b); however, the control monkeys-but not the vaccinated monkeys-responded with an increase in neutralizing-antibody titres (Extended Data Fig. 4c), consistent with reports that SARS-CoV-2 infection of rhesus macaques results in mild disease ${ }^{19,20}$.

Vaccine-associated enhanced respiratory disease (VAERD) has previously been described for respiratory infections with respiratory syncytial virus and SARS-CoV ${ }^{21,22}$. We evaluated inflammation in the lung tissues of a subset of the tested monkeys using positron emission tomography with computed tomography (PET-CT) on the day of the challenge and four to five days after challenge. Of the 6 monkeys we evaluated ( 2 unvaccinated, 2 from the ASO3 group and 2 from the $\mathrm{CpG}$-alum group, selected randomly), we found inflammation in both control monkeys on day 4 compared with baseline, as measured by enhanced 2-deoxy-2-[ $\left.{ }^{18} \mathrm{~F}\right]$ fluoroglucose (FDG) uptake. By contrast, only one of the four vaccinated monkeys showed FDG uptake, to a much lower extent than the control monkeys (Extended Data Fig. 4d, e). Additionally, we performed a comprehensive analysis of cytokine responses in all the monkeys one week after challenge, and observed no increase in inflammation in the lungs of the vaccinated monkeys (Extended Data Fig. 5a, b), whereas there were increases of cytokines such as IL-6, IL-8, IFN $\gamma$ and MCP4 in the lungs of control monkeys (Extended Data Fig. 5c). These data are consistent with an absence of VAERD in these monkeys. However, these results should be interpreted with caution, as VAERD is expected to occur as immunity decreases after immunization.

\section{Immune correlates of protection}

Next, we correlated humoral and cellular immune responses measured at peak time points (day 42 for antibody responses and day 28 for T cell responses) with the viral load (nasal or pharyngeal) to determine 


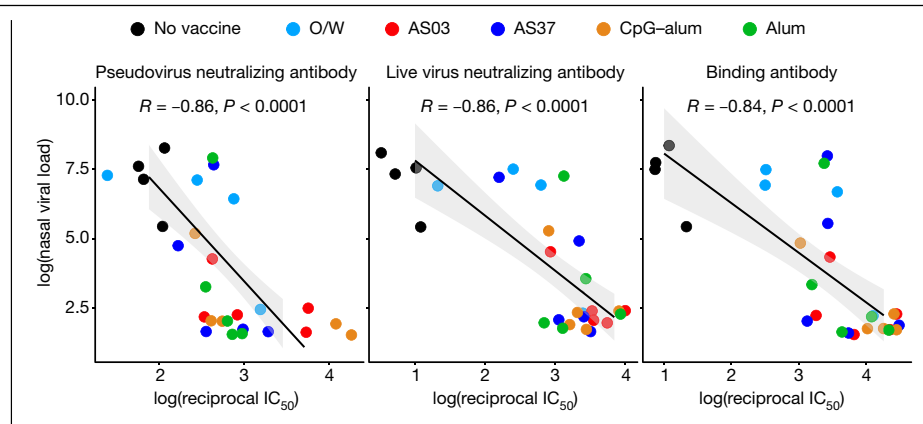

Fig. 4 | Immune correlates of protection. Spearman's correlation between peak nasal viral load and neutralizing and binding-antibody responses, the top three parameters from the correlates analysis shown in Extended Data Fig. 6a. Shaded areas represent $95 \%$ confidence intervals. Each symbol represents a monkey. $n=4$ (control and $\mathrm{O} / \mathrm{W}$ ) and 5 (all other groups).

the putative correlates of protection in an unbiased approach. Neutralizing titres for both live virus and pseudovirus emerged as the top statistically significant correlates of protection (Fig. 4, Extended Data Fig. 6a, b) in both nasal and pharyngeal compartments. Of note, the nanoparticle-specific IL-2 ${ }^{+} \mathrm{TNF}^{+} \mathrm{CD} 4 \mathrm{~T}$ cell response also emerged as a significant correlate of protection in both compartments (Extended Data Fig. $6 a-c)$, the frequencies of which positively correlated with neutralizing-antibody titres (Extended Data Fig. 6d). This is consistent with the possibility that nanoparticle-specific CD4 T cells could offer T cell help to RBD-specific $B$ cells.

In addition to characterizing neutralizing-antibody and T cell responses to vaccination, we sought to understand the humoral functional profile elicited by each adjuvant. Vaccines rapidly induced an increase in different anti-spike antibody isotypes (Extended Data Fig. 7a-c), Fc-receptor-binding (Extended Data Fig. 7d) and antibody-dependent neutrophil phagocytosis (ADNP) (Extended Data Fig. 7e) at day 21 and day 42. To understand how differences in the humoral response could lead to viral breakthrough, we performed a partial least-squares discriminant analysis (PLSDA) on the antibody features measured at day 42, using least absolute shrinkage and selection operator (LASSO) to select features to prevent overfitting (Extended Data Fig. 7f). The PLSDA analysis showed separation between protected and infected monkeys (Extended Data Fig. 7f), marked by an enrichment in IgA, FcR3A and ADNP in the protected monkeys (Extended Data Fig. 7g). Next, we determined the correlation of each measured antibody feature and the peak nasal and pharyngeal viral load to further dissect the antibody features that provide protection against viral break-through. Although the neutralizing-antibody response still represented the strongest correlate of protection, we observed additional functional features, including FcR binding and ADNP, that were negatively correlated with nasal or pharyngeal viral loads (Extended Data Fig. 7h). These data demonstrated an additive role for functional antibody responses in protection. Furthermore, each adjuvant group mounted a distinct profile of antibody response that correlated with protection against the virus (Extended Data Fig. 7i).

\section{RBD-NP versus prefusion spike HexaPro}

The data described thus far demonstrate that RBD-NP immunogen when adjuvanted with AS03, AS37, CpG-alum or alum induces robust protective immunity. Next, we compared the immunogenicity of the RBD-NP immunogen to that of HexaPro, a highly stable variant of the prefusion spike trimer ${ }^{23}$, in either soluble form or attached to a nanoparticle (HexaPro-NP, with 20 HexaPro trimers displayed on the I53-50 nanoparticle). We immunized three cohorts of rhesus macaques with RBD-NP, soluble HexaPro or HexaPro-NP, each with AS03 as adjuvant (Extended Data Fig. 8a, Extended Data Table 1). The RBD-NP-AS03
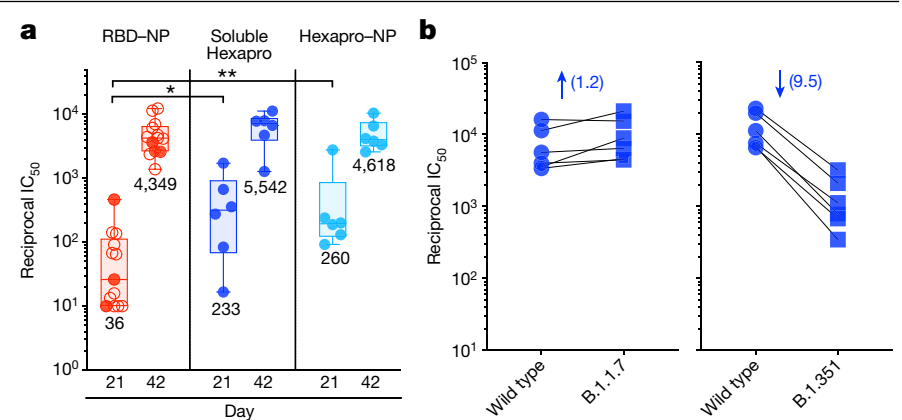

Fig. 5 | RBD-NP or HexaPro immunization with ASO3 elicit similar neutralizing-antibody responses. a, Neutralizing-antibody responses in serum on day 21 and day 42 against SARS-CoV-2 virus. The box plot shows median, 25th and 75th percentiles and the whiskers show the range. Numbers indicate GMT. Statistically significant differences between two groups by two-sided Mann-Whitney rank-sum test. ${ }^{*} P=0.02,{ }^{* *} P=0.006$. Open circles denote monkeys from the earlier study shown in Fig. 1.b, Neutralizing-antibody titres measured against live wild-type (circles) or B.1.1.7 or B.1.351 variants (squares) in sera collected on day 42 from monkeys that received soluble HexaPro. Each symbol represents one monkey. $n=13$ for RBD-NP and 6 for soluble HexaPro and HexaPro-NP groups.

immunization induced neutralizing-antibody titres consistent with those described in Fig. 1, with a detectable titre on day 21 and a robust increase on day 42. Compared with RBD-NP immunization, immunization with soluble HexaPro or HexaPro-NP induced notably higher neutralizing-antibody titres after a single immunization. However, the neutralizing-antibody titres on day 42 were similar in all three groups (Fig. 5a, Extended Data Fig. 8b). Furthermore, HexaPro-AS03 immunization also elicited cross-reactive neutralizing responses against the SARS-CoV-2 B.1.1.7 and B.1.351 variants (Fig. 5b), similar to the response to RBD-NP immunization (Fig. 1d). Together, these data indicate that the RBD-NP is as potent an immunogen as the highly stable HexaPro, consistent with previous observations that the vast majority of the neutralizing response targets the $\mathrm{RBD}^{12}$. Moreover, these data also suggest that ASO3 may be a suitable adjuvant for clinical use with various forms of the spike protein.

\section{Discussion}

Despite the deployment of several million doses of vaccines worldwide ${ }^{8,24-26}$, only a small fraction of the global population has been vaccinated to date. There remains a stark gap between the vaccination rates in different countries, with many developing countries yet to report a single dose. Furthermore, specific subpopulations such as infants and the elderly could benefit from the use of adjuvanted subunit vaccine platforms with a demonstrable history of safety and efficacy in such populations ${ }^{27,28}$. Here, we have evaluated five adjuvants, and all five induced substantial neutralizing-antibody titres. $\mathrm{O} / \mathrm{W}$ induced relatively lower neutralizing titres than the other adjuvants; although $\mathrm{O} / \mathrm{W}$ is an oil-in-water emulsion similar to AS03, it does not contain $\alpha$-tocopherol, which is thought to be required for the potent antibody responses observed with $\mathrm{ASO}^{29}$. Notably, there were also potent CD4 $\mathrm{T}$ cell responses specific to the nanoparticle scaffold.

The different adjuvants produced varying levels of protection against SARS-CoV-2. The neutralizing response was the primary correlate of protection, as previously seen ${ }^{10,16}$. However, the frequency of nanoparticle-specific IL- ${ }^{+} \mathrm{TNF}^{+}$cells were also correlated with protection and neutralizing-antibody titres (Extended Data Fig. 6c,d). Thus, these nanoparticle-scaffold-specific CD4 T cells may provide $\mathrm{T}$ cell help to RBD-specific B cells, thereby promoting B cell responses ${ }^{30}$. In addition, the $T$ cells could provide a complementary mechanism of protection that synergizes with the neutralizing-antibody response ${ }^{31}$. 


\section{Article}

The adjuvants also induced different $\mathrm{T}_{\mathrm{H}} 1-\mathrm{T}_{\mathrm{H}} 2$ profiles, with RBDNP-ASO3 stimulating a mixed $\mathrm{T}_{\mathrm{H}} 1-\mathrm{T}_{\mathrm{H}} 2$ response, whereas RBDNP-CpG-alum and RBD-NP-AS37 stimulated a $\mathrm{T}_{\mathrm{H}} 1$-biased response and RBD-NP-alum and RBD-NP-O/W induced $\mathrm{T}_{\mathrm{H}} 2$-biased responses. We saw no evidence of VAERD in the challenged monkeys.

In addition to evaluating clinically relevant adjuvants, we also compared the immunogenicity of RBD and HexaPro. Our results demonstrate that the RBD-NP immunogen was as potent as HexaPro in inducing neutralizing-antibody titres. Whether differences in immunogenicity become apparent at lower doses of antigen warrants further investigation. Nevertheless, these results demonstrate that ASO3 is a potent adjuvant when used with either RBD-NP or HexaPro. It will be of particular interest to the field to evaluate whether the neutralizing responses elicited by RBD-NP or HexaPro-based immunogens induce a broad response not only against the emerging SARS-CoV-2 variants, but also against other coronaviruses.

In summary, this study represents a comprehensive immunological benchmarking of clinically relevant adjuvants for their capacity to enhance the protective immunity of a SARS-CoV-2 vaccine. On the basis of these results, two phase I/II clinical trials (NCT04742738 and NCT04750343) have been initiated by SK Bioscience in collaboration with Coalition for Epidemic Preparedness Innovations (CEPI) for the development of COVID-19 vaccines.

\section{Online content}

Any methods, additional references, Nature Research reporting summaries, source data, extended data, supplementary information, acknowledgements, peer review information; details of author contributions and competing interests; and statements of data and code availability are available at https://doi.org/10.1038/s41586-021-03530-2.

1. Krammer, F. SARS-CoV-2 vaccines in development. Nature 586, 516-527 (2020).

2. Pollard, A. J. \& Bijker, E. M. A guide to vaccinology: from basic principles to new developments. Nat. Rev. Immunol. 21, 83-100 (2021).

3. Pulendran, B., S Arunachalam, P. \& O'Hagan, D. T. Emerging concepts in the science of vaccine adjuvants. Nat. Rev. Drug Discov. https://doi.org/10.1038/s41573-021-00163-y (2021).

4. Thanh Le, T. et al. The COVID-19 vaccine development landscape. Nat. Rev. Drug Discov. 19, 305-306 (2020).

5. Walls, A. C. et al. Elicitation of potent neutralizing antibody responses by designed protein nanoparticle vaccines for SARS-CoV-2. Cell 183, 1367-1382 (2020).

6. Walls, A. C. et al. Structure, function, and antigenicity of the SARS-CoV-2 spike glycoprotein. Cell 181, 281-292 (2020).

7. Suthar, M. S. et al. Rapid generation of neutralizing antibody responses in COVID-19 patients. Cell Rep. Med. 1, 100040 (2020).

8. Anderson, E. J. et al. Safety and immunogenicity of SARS-CoV-2 mRNA-1273 vaccine in older adults. N. Engl. J. Med. 383, 2427-2438 (2020).

9. Widge, A. T. et al. Durability of responses after SARS-CoV-2 mRNA-1273 vaccination. N. Engl. J. Med. 384, 80-82 (2021).

10. Mercado, N. B. et al. Single-shot Ad26 vaccine protects against SARS-CoV-2 in rhesus macaques. Nature 586, 583-588 (2020).

11. Case, J. B. et al. Neutralizing antibody and soluble ACE2 inhibition of a replication-competent VSV-SARS-CoV-2 and a clinical isolate of SARS-CoV-2. Cell Host Microbe 28, 475-485 (2020).

12. Piccoli, L. et al. Mapping neutralizing and immunodominant sites on the SARS-CoV-2 spike receptor-binding domain by structure-guided high-resolution serology. Cell 183 1024-1042 (2020).
13. Wu, K. et al. Serum neutralizing activity elicited by mRNA-1273 vaccine-preliminary report. N. Engl. J. Med. 384, 1468-1470 (2021).

14. Liu, Y. et al. Neutralizing activity of BNT162b2-elicited serum. N. Engl. J. Med. 384, 1466-1468 (2021).

15. Francica, J. R. et al. Innate transcriptional effects by adjuvants on the magnitude quality, and durability of HIV envelope responses in NHPs. Blood Adv. 1, 2329-2342 (2017).

16. Corbett, K. S. et al. Evaluation of the mRNA-1273 vaccine against SARS-CoV-2 in nonhuman primates. N. Engl. J. Med. 383, 1544-1555 (2020).

17. van Doremalen, $\mathrm{N}$. et al. ChAdOx $1 \mathrm{nCoV}-19$ vaccine prevents SARS-CoV-2 pneumonia in rhesus macaques. Nature 586, 578-582 (2020).

18. Kim, D. et al. The architecture of SARS-CoV-2 transcriptome. Cell 181, 914-921 (2020).

19. Hewitt, J. A. et al. ACTIVating resources for the COVID-19 pandemic: in vivo models for vaccines and therapeutics. Cell Host Microbe 28, 646-659 (2020).

20. Vogel, A. B. et al. BNT162b vaccines protect rhesus macaques from SARS-CoV-2. Nature 592, 283-289 (2021).

21. Acosta, P. L., Caballero, M. T. \& Polack, F. P. Brief history and characterization of enhanced respiratory syncytial virus disease. Clin. Vaccine Immunol. 23, 189-195 (2015).

22. Bolles, M. et al. A double-inactivated severe acute respiratory syndrome coronavirus vaccine provides incomplete protection in mice and induces increased eosinophilic proinflammatory pulmonary response upon challenge. J. Virol. 85, 12201-12215 (2011).

23. Hsieh, C. L. et al. Structure-based design of prefusion-stabilized SARS-CoV-2 spikes. Science 369, 1501-1505 (2020).

24. Sadoff, J. et al. Interim results of a phase 1-2a trial of Ad26.COV2.S Covid-19 vaccine. N. Engl. J. Med. https://doi.org/10.1056/NEJMoa2034201 (2021)

25. Voysey, M. et al. Safety and efficacy of the ChAdOx1 nCoV-19 vaccine (AZD1222) against SARS-CoV-2: an interim analysis of four randomised controlled trials in Brazil, South Africa, and the UK. Lancet 397, 99-111 (2021).

26. Walsh, E. E. et al. Safety and immunogenicity of two RNA-based Covid-19 vaccine candidates. N. Engl. J. Med. 383, 2439-2450 (2020).

27. Doherty, M. et al. Vaccination of special populations: Protecting the vulnerable. Vaccine 34, 6681-6690 (2016)

28. Cohet, C. et al. Safety of ASO3-adjuvanted influenza vaccines: A review of the evidence. Vaccine 37, 3006-3021 (2019).

29. Morel, S. et al. Adjuvant system ASO3 containing a-tocopherol modulates innate immune response and leads to improved adaptive immunity. Vaccine 29, 2461-2473 (2011).

30. Brouwer, P. J. M. et al. Two-component spike nanoparticle vaccine protects macaques from SARS-CoV-2 infection. Cell 184, 1188-1200 (2021).

31. Arunachalam, P. S. et al. T cell-inducing vaccine durably prevents mucosal SHIV infection even with lower neutralizing antibody titers. Nat. Med. 26, 932-940 (2020).

Publisher's note Springer Nature remains neutral with regard to jurisdictional claims in published maps and institutional affiliations.

(c) The Author(s), under exclusive licence to Springer Nature Limited 2021

${ }^{1}$ Institute for Immunity, Transplantation and Infection, Stanford University School of Medicine, Stanford University, Stanford, CA, USA. ${ }^{2}$ Department of Biochemistry, University of Washington, Seattle, WA, USA. ${ }^{3}$ Tulane National Primate Research Center, Covington, LA, USA. ${ }^{4}$ Ragon Institute of MIT, MGH and Harvard, Cambridge, MA, USA. ${ }^{5}$ Yerkes National Primate Research Center, Atlanta, Georgia, USA. ${ }^{6}$ Department of Pathology, Stanford University School of Medicine, Stanford University, Stanford, CA, USA. ${ }^{7}$ New Iberia Research Center, University of Louisiana at Lafayette, New Iberia, LA, USA. ${ }^{8}$ Institute for Protein Design, University of Washington, Seattle, WA, USA. ${ }^{9}$ Department of Microbiology and Molecular Genetics, University of Pittsburgh School of Medicine, Pittsburgh, PA, USA. ${ }^{10} \mathrm{Center}$ for Vaccine Research, University of Pittsburgh School of Medicine, Pittsburgh, PA, USA. ${ }^{11}$ Department of Molecular Biosciences, University of Texas, Austin, TX, USA. ${ }^{12}$ World Reference Center for Emerging Viruses and Arboviruses, University of Texas Medical Branch, Galveston, TX, USA. ${ }^{13}$ Department of Surgery, Duke University School of Medicine, Durham, NC, USA. ${ }^{14}$ Department of Bioengineering, University of California, San Diego, La Jolla, CA, USA. ${ }^{15} \mathrm{GSK}$, Rockville, MD, USA. ${ }^{16} \mathrm{GSK}$, Rixensart, Belgium. ${ }^{17} \mathrm{GSK}$, Siena, Italy. ${ }^{18}$ Dynavax Technologies Corporation, Emeryville, CA, USA. ${ }^{19}$ Bill and Melinda Gates Foundation, Seattle, WA, USA. ${ }^{20}$ Department of Microbiology and Immunology, Tulane University School of Medicine, New Orleans, LA, USA. ${ }^{21}$ Department of Microbiology and Immunology, Stanford University School of Medicine, Stanford University, Stanford, CA, USA. ${ }^{\bowtie}$-mail: bpulend@ stanford.edu 


\section{Methods}

No statistical methods were used to predetermine sample size. The experiments were not randomized. The investigators were not blinded to allocation during experiments and outcome assessment.

\section{Animal subjects and experimentation}

Thirty-three male rhesus macaques (Macaca mulatta) of Indian origin, aged 3-9 years, were assigned to the study (Extended Data Table 1). The animals were distributed between the groups such that the age and weight distribution were comparable across the groups. Animals were housed and maintained as per National Institutes of Health (NIH) guidelines at the New Iberia Research Center (NIRC) of the University of Louisiana at Lafayette in accordance with the rules and regulations of the Committee on the Care and Use of Laboratory Animal Resources. The entire study (protocol 2020-8808-15) was reviewed and approved by the University of Louisiana at Lafayette Institutional Animal Care and Use Committee (IACUC). All animals were negative for simian immunodeficiency virus, simian $\mathrm{T}$ cell leukaemia virus and simian retrovirus. For the challenge, the animals were transferred to the Regional Biosafety Level 3 facility at the Tulane National Primate Research Center, where the study was reviewed and approved by the Tulane University IACUC (protocol3918).

\section{RBD-16GS-I53-50 nanoparticle immunogen production}

Nanoparticle immunogen components and nanoparticles were produced as previously described in detail ${ }^{5}$, with the exception that the nanoparticle was in a buffer containing $50 \mathrm{mM}$ Tris $\mathrm{pH} 8,150 \mathrm{mM} \mathrm{NaCl}$, $100 \mathrm{mM} \mathrm{L}$-arginine and 5\% sucrose.

\section{Nanoparticle biochemical characterization}

Dynamic light scattering, negative stain electron microscopy, and maACE2-Fc and CR3022 IgG biolayer interferometry were performed as described previously.

\section{Adjuvant formulations and immunization}

Essai O/W1849101, a squalene-in-water emulsion (O/W) was provided by Seppic. For each dose, RBD-NP was diluted to $50 \mathrm{\mu g} \mathrm{ml}^{-1}$ (RBD component) in $250 \mu$ l of phosphate buffered saline (PBS) with $150 \mathrm{mM} \mathrm{NaCl}$ and mixed with an equal volume of O/W. The dose of O/W was $50 \%(\mathrm{v} / \mathrm{v})$ AS03 and AS37 were provided by GSK Vaccines. ASO3 is an oil-in-water emulsion that contains $11.86 \mathrm{mg} \alpha$-tocopherol, $10.69 \mathrm{mg}$ squalene, and $4.86 \mathrm{mg}$ polysorbate 80 (Tween-80) in PBS, whereas AS37 is a TLR-7 agonist $\left(200 \mu \mathrm{g} \mathrm{ml}^{-1}\right)$ adsorbed to aluminium hydroxide $\left(2 \mathrm{mg} \mathrm{ml}^{-1}\right)$. For each dose, RBD-NP was diluted to $50 \mathrm{\mu g} \mathrm{ml}^{-1}$ (RBD component) in $250 \mu$ lof Tris-buffered saline (TBS) and mixed with an equal volume of AS03 or AS37. The dose of AS03 was 50\% (v/v) (equivalent of one human dose), AS37 included $50 \mu \mathrm{g}$ TLR-7 agonist and $0.5 \mathrm{mg}$ aluminium hydroxide. $\mathrm{CpG} 1018$ was provided by Dynavax Technologies at a concentration of $12 \mathrm{mg} \mathrm{ml}^{-1}$. Alum (Alhydrogel 2\%) was purchased from Croda Healthcare (batch 0001610348). Of note, we used CpG-alum rather than $\mathrm{CpG} 1018$ (no alum), which is used in Heplisav-B. For each dose of CpG-alum, $25 \mu$ g antigen (RBD component) in TBS was mixed with $0.75 \mathrm{mg}$ alum and incubated on ice for $30 \mathrm{~min}$. After 30 min of incubation, $1.5 \mathrm{mg}$ of $\mathrm{CpG} 1018$ was added and mixed rapidly. Each dose contained $1.5 \mathrm{mg} \mathrm{CpG} 1018$ and $0.75 \mathrm{mg}$ alum. For each dose of alum, $25 \mu \mathrm{g}$ antigen (RBD component) in TBS was mixed with $0.75 \mathrm{mg}$ alum, matching the concentration of alum in the $\mathrm{CpG}$-alum formulation, and incubated on ice for $30 \mathrm{~min}$. Soluble HexaPro or HexaPro-NP used in the experiment to compare RBD-NPversus HexapPro (described in Fig. 5) was diluted to $50 \mu \mathrm{g} \mathrm{ml}^{-1}$ in $250 \mu \mathrm{l} \mathrm{TBS}$ and mixed with an equal volume of AS03. All immunizations were administered via the intramuscular route in right forelimbs. The volume of each dose was $0.5 \mathrm{ml}$.

\section{Anti-spike binding ELISA}

SARS-CoV-2 spike protein was produced in HEK 293T cells (Atum). Ninety-six-well Corning Costar high-binding plates (Thermo Fisher
Scientific) were coated with SARS-CoV-2 spike protein in PBS at a concentration of $0.2 \mu \mathrm{g}$ per well overnight at $4{ }^{\circ} \mathrm{C}$. On the next day, wells were washed 3 times with PBS, 0.1\% Tween 20 (PBS-T) and blocked with PBS-T containing 3\% non-fat milk powder for $1 \mathrm{~h}$ at room temperature. Wells were then incubated with plasma samples from non-human primates at different dilutions starting at 1:100 in PBS-T containing $1 \%$ non-fat milk for $1 \mathrm{~h}$ at $37^{\circ} \mathrm{C}$. After washing 3 times with PBS-T, horseradish peroxidase (HRP)-conjugated goat anti-monkey IgG ( $\gamma$-chain specific, Alpha Diagnostics, 1:4,000 dilution), in PBS-T containing $1 \%$ non-fat milk was added and incubated for $1 \mathrm{~h}$ at room temperature. Wells were washed 3 times with PBS-T before addition of 3,3',5,5'-tetramethylbenzidine (TMB) substrate solution. The reaction was stopped after $12 \mathrm{~min}$ by addition of $0.16 \mathrm{M}$ sulfuric acid. The optical density (OD) at 450 nanometers was measured with a Tecan Infinite M Nano Plus microplate reader.

\section{Anti-153-50 ELISA}

The protocol was adapted from Tiller et al..$^{32}$. In brief, recombinant I5350 protein nanoparticles, SARS-CoV-2S2P trimers, or goat anti-human IgG (Jackson ImmunoResearch, 109-005-044) were immobilized on 96-well Nunc MaxiSorp (Thermo Fisher Scientific) plates $\left(2 \mu \mathrm{g} \mathrm{ml}^{-1}\right.$, $50 \mu \mathrm{l}$ per well). After $1 \mathrm{~h}$ incubation at room temperature, plates were blocked with $200 \mu \mathrm{l} \mathrm{TBS,} 2 \%(\mathrm{w} / \mathrm{v})$ BSA and $0.05 \%(\mathrm{v} / \mathrm{v})$ Tween 20 for $1 \mathrm{~h}$. Plates were washed 3 times in TBST with a plate washer (BioTek), and $50 \mu \mathrm{l}$ of $1: 5$ serial dilutions starting at 1:100 of non-human primate sera in TBST incubated for $1 \mathrm{~h}$ in wells with I53-50 or spike protein. In wells with anti-human IgG capture antibody, human IgG control (SinoBiological, $\mathrm{HG1K}$ ) was serially diluted from $0.5-500 \mathrm{ng} \mathrm{ml}^{-1}$ in TBST in triplicate and $50 \mu \mathrm{l}$ of each dilution incubated for $1 \mathrm{~h}$. Plates were washed 3 times in TBST, then HRP-conjugated goat anti-monkey IgG (Alpha Diagnostics, 70021) was diluted $1: 5,000$ in $2 \%$ BSA in TBST and $50 \mu$ was incubated in each well for $30 \mathrm{~min}$. Plates were washed 3 times in TBST and $100 \mu \mathrm{l}$ TMB (SeraCare) was added to each well for $2 \mathrm{~min}$. The reaction was quenched by adding $100 \mu \mathrm{l}$ of $1 \mathrm{~N} \mathrm{HCl}$. Plates were immediately read at $450 \mathrm{~nm}$ on a SpectraMax M5 plate reader (Molecular Devices). The data were plotted with GraphPad Prism. A sigmoidal 4PL curve was fit with $x$ being $\log$ (concentration) to determine the $\mathrm{EC}_{50}$ values. A logarithmic equation fit to the linear portion of the sigmoidal curve of the human IgG control was used to calculate concentration (in $\mathrm{mg} \mathrm{ml}^{-1}$ ) of IgG in sera for anti-153-50 and anti-spike titres. All steps were performed at ambient temperature.

\section{Pseudovirus production and neutralization assay}

Pseudovirus production has been described in Walls et al. ${ }^{6}$. In brief, MLV-based SARS-CoV-2 spike pseudotyped viruses were prepared as previously ${ }^{6,33,34}$ except that the SARS-CoV-2 spike construct contained the D614G mutation and a truncation of the $\mathrm{C}$-terminal 21 residues $^{11,35}$.

For neutralization assays, HEK-hACE2 cells were cultured in DMEM with $10 \% \mathrm{FBS}$ (Hyclone) and $1 \%$ penicillin-streptomycin with $8 \% \mathrm{CO}_{2}$ in a $37^{\circ} \mathrm{C}$ incubator on 96 well plates coated with poly-L-lysine (Sigma). To coat plates, $40 \mu \mathrm{l}$ poly-L-lysine (Sigma) was incubated with rotation for $5 \mathrm{~min}$. Poly-L-lysine was removed, plates were dried for $5 \mathrm{~min}$ then washed once with water before plating cells. The following day, cells were checked to be at $80 \%$ confluence. In a half-area 96 -well plate a 1:3 serial dilution of sera was made in DMEM in $22 \mu \mathrm{l}$ final volume. Twenty-two microlitres of pseudovirus was then added to the serial dilution and incubated at room temperature for 30-60 min at room temperature. The medium on the HEK-hACE2 plate was removed and $40 \mu \mathrm{l}$ of the sera-virus mixture was added to the cells and incubated for $2 \mathrm{~h}$ at $37^{\circ} \mathrm{C}$ with $8 \% \mathrm{CO}_{2}$. Following incubation, $40 \mu \mathrm{l} 20 \% \mathrm{FBS}$ and $2 \%$ PenStrep containing DMEM was added to the cells. Following $48-72 \mathrm{~h}$ infection, One-Glo-EX (Promega) was added to the cells in half culturing volume ( $40 \mu \mathrm{l}$ added) and incubated in the dark for $5 \mathrm{~min}$ before reading on a Varioskan LUX plate reader (Thermo Fisher Scientific). 
Measurements were performed on all sera samples from each group in at least duplicates. Relative luciferase units were plotted and normalized in Prism (GraphPad) using a zero value of cells alone and a 100\% value of 1:2 virus alone. Nonlinear regression of $\log$ (inhibitor) versus normalized response was used to determine $\mathrm{IC}_{50}$ values from curve fits. The human convalescent samples assayed in parallel were obtained from individuals aged 37-67 years, all of whom had mild-to-moderate disease with fever, cough, chills, shivering, runny nose, muscle aches, trouble breathing and fatigue as symptoms. The use of samples was approved by the University of Washington Human Subjects Division Institutional Review Board (IRB00009810).

\section{FRNT assay}

Neutralization assays with authentic SARS-CoV-2 virus were performed as previously described ${ }^{36}$. Plasma or serum were serially diluted (threefold) in serum-free Dulbecco's modified Eagle's medium (DMEM) in duplicate wells and incubated with 100-200 focus forming units (FFU) infectious clone-derived SARS-CoV-2-mNG virus ${ }^{37}$ at $37^{\circ} \mathrm{C}$ for $1 \mathrm{~h}$. The antibody-virus mixture was added to VeroE6 cell (C1008, ATCC, CRL1586) monolayers seeded in 96-well blackout plates and incubated at $37^{\circ} \mathrm{C}$ for $1 \mathrm{~h}$. After incubation, the inoculum was removed and replaced with pre-warmed complete DMEM containing $0.85 \%$ methylcellulose. Plates were incubated at $37^{\circ} \mathrm{C}$ for $24 \mathrm{~h}$. After $24 \mathrm{~h}$, methylcellulose overlay was removed, cells were washed twice with PBS and fixed with $2 \%$ paraformaldehyde in PBS for 30 min at room temperature. Following fixation, plates were washed twice with PBS and foci were visualized on a fluorescence ELISPOT reader (CTL ImmunoSpot S6 Universal Analyzer) and counted using Viridot ${ }^{38}$. The neutralization titres were calculated as follows: 1 - (mean number of foci in the presence of sera/ number of foci at the highest dilution of the respective serum sample). Each specimen was tested in two independent assays performed at different times. The FRNT- $\mathrm{mNG}_{50}$ titres were interpolated using a 4-parameter nonlinear regression in GraphPad Prism 8.4.3. Samples with an FRNT- $\mathrm{mNG}_{50}$ value that was below the limit of detection were plotted at 10 . For these samples, this value was used in fold-reduction calculations.

\section{ACE2 blocking assay}

Antibodies blocking the binding of SARS-CoV-2 spike RBD to ACE2 were detected with a V-PLEXSARS-CoV-2 Panel 2 (ACE2) Kit (Meso Scale Diagnostics) according to the manufacturer's instructions. Serum samples from non-human primates were analysed in duplicate at a dilution of 1:100 and per cent inhibition was calculated as (1-average sample ECL signal/average ECL signal of calibrator 7) $\times 100$.

\section{Pseudovirus neutralization assay against UK B.1.1.7 variant}

A neutralization assay comparing the ability of sera from vaccinated animals to neutralize wild-type (with D614G in spike) SARS-CoV-2 versus the B.1.1.7 variant were performed using a pseudotyped virus-neutralization assay as previously reported ${ }^{39}$, with minor modifications. In brief, mutations were introduced into a plasmid expressing codon-optimized spike of the Wuhan-1 strain that contains the D614G mutation using site-directed mutagenesis. Pseudovirions were produced in HEK 293T/17 cells by co-transfection of a lentivirus backbone plasmid, a spike-expressing plasmid, and a firefly luciferase reporter gene plasmid. Pseudotyped viruses were titrated in 293T/ ACE2.MF cells for median tissue culture infectious dose (TCID50) and used for neutralization assay. Viruses were incubated with serial diluted serum samples at $37^{\circ} \mathrm{C}$ for $1 \mathrm{~h}$, and subsequently added to cells and incubated for 66-72 h. Luminescence was measured using a GloMax Navigator luminometer (Promega). Neutralization titres are the inhibitory dilution (ID) of serum samples at which relative luminescence unit $(\mathrm{RLU})$ readings were reduced by either $50 \%\left(\mathrm{ID}_{50}\right)$ or $80 \%\left(\mathrm{ID}_{80}\right)$ compared to virus control wells after subtraction of background RLUs.

\section{FRNT assay against the variants of concern}

The wild-type infectious clone SARS-CoV-2 (icSARS-CoV-2), derived from the 2019-nCoV/USA_WA1/2020 strain, was propagated in VeroE6 cells (ATCC) and sequenced ${ }^{37}$. The B.1.1.7 variant (SARS-CoV-2/human/ USA/CA_CDC_5574/2020) was isolated from a residual nasopharyngeal swab collected from a patient in San Diego, CA, propagated in Vero cells and sequenced. The RSA B.1.351 variant was isolated as previously described $^{40}$. Our laboratory plaque-isolated the virus on VeroE6 cells followed by a single round of propagation on VeroE6 cells (multiplicity of infection 0.05), aliquoted to generate a working stock and sequenced. Viral titres were determined by focus-forming assay on VeroE6 cells. Viral stocks were stored at $-80^{\circ} \mathrm{C}$ until use.

FRNT assays were performed as previously described for the wild-type FRNT assay. The assay with each variant was performed simultaneously with wild-type controls. The samples were diluted 3-fold in 8 serial dilutions using DMEM in duplicates with an initial dilution of 1:10 in a total volume of $60 \mu$ l. Serially diluted samples were incubated with an equal volume of wild-type or variant SARS-CoV-2 (100-200 foci per well) at $37^{\circ} \mathrm{C}$ for $1 \mathrm{~h}$ in a round-bottomed 96 -well culture plate. The antibody-virus mixture was then added to Vero cells and incubated at $37^{\circ} \mathrm{C}$ for $1 \mathrm{~h}$. After incubation, the antibodyvirus mixture was removed and $100 \mu$ l of prewarmed $0.85 \%$ overlay was added to each well. Plates were incubated at $37^{\circ} \mathrm{C}$ for $24 \mathrm{~h}$. After $24 \mathrm{~h}$, methylcellulose overlay was removed, and cells were washed 3 times with PBS. Cells were then fixed with $2 \%$ paraformaldehyde in PBS (Electron Microscopy Sciences) for $30 \mathrm{~min}$. Following fixation, plates were washed twice with PBS and $100 \mu$ l of permeabilization buffer (0.1\% BSA, saponin in PBS), was added to the fixed Vero cells for $20 \mathrm{~min}$. Cells were incubated with an anti-SARS-CoV spike primary antibody directly conjugated to biotin (CR3022-biotin) for $1 \mathrm{~h}$ at room temperature. Next, the cells were washed three times in PBS and avidin-HRP was added for $1 \mathrm{~h}$ at room temperature followed by three washes in PBS. Foci were visualized using TrueBlue HRP substrate (KPL, 5510-0050) and imaged on an ELISPOT reader (CTL).

\section{Intracellular cytokine staining assay}

Antigen-specific T cell responses were measured using the intracellular cytokine staining assay. Live frozen PBMCs were revived, counted and resuspended at a density of $10^{6}$ live cells per $\mathrm{ml}$ in complete RPMI (RPMI supplemented with $10 \%$ FBS and antibiotics). The cells were rested overnight at $37^{\circ} \mathrm{C}$ in a $\mathrm{CO}_{2}$ incubator. Next morning, the cells were counted again, resuspended at a density of $15 \times 10^{6}$ per $\mathrm{ml}$ in complete RPMI and $100 \mu \mathrm{l}$ of cell suspension containing $1.5 \times 10^{6}$ cells was added to each well of a 96-well round-bottomed tissue culture plate. Each sample was treated with three conditions, no stimulation, a peptide pool spanning the RBD region of spike at a concentration of $1.2 \mathrm{\mu g} \mathrm{ml}^{-1}$ of each peptide and a peptide pool spanning the I53-50A, and I53-50B components of the nanoparticle scaffold $\left(1.2 \mu \mathrm{g} \mathrm{ml}^{-1}\right.$ of each peptide) in the presence of $1 \mu \mathrm{g} \mathrm{ml}^{-1}$ of anti-CD28 (clone CD28.2, BD Biosciences) and anti-CD49d (clone 9F10, BD Biosciences) as well as anti-CXCR3 and anti-CXCR5. The peptides were custom synthesized to $90 \%$ purity using GenScript, a commercial vendor. All samples contained $0.5 \%(\mathrm{v} / \mathrm{v})$ DMSO in total volume of $200 \mu \mathrm{l}$ per well. The samples were incubated at $37^{\circ} \mathrm{C}$ in $\mathrm{CO} 2$ incubators for $2 \mathrm{~h}$ before addition of $10 \mu \mathrm{g} \mathrm{ml}^{-1}$ brefeldin A. The cells were incubated for an additional $4 \mathrm{~h}$. The cells were washed with PBS and stained with Zombie UV fixable viability dye (Biolegend). The cells were washed with PBS containing $5 \% \mathrm{FCS}$, before the addition of surface antibody cocktail. The cells were stained for $20 \mathrm{~min}$ at $4{ }^{\circ} \mathrm{C}$ in $100 \mu$ volume. Subsequently, the cells were washed, fixed and permeabilized with cytofix/cytoperm buffer (BD Biosciences) for $20 \mathrm{~min}$. The permeabilized cells were stained with intracellular cytokine staining antibodies for $20 \mathrm{~min}$ at room temperature in $1 \times$ perm/wash buffer (BD Biosciences). Cells were then washed twice with perm/wash buffer and once with staining buffer before acquisition using the BD Symphony 
Flow Cytometer and the associated BD FACS Diva software. All flow cytometry data were analysed using Flowjo software v10 (TreeStar Inc.).

\section{Viral challenge}

Animals were inoculated via the intratracheal and intranasal routes with a total of $3.2 \times 10^{6}$ plaque-forming units of SARS-CoV-2, isolate USA WA1/2020 (accession: MN985325). The virus stock was generated by expansion of a seed stock on Vero E6 cells and titred by plaque assay on Vero E6 cells. It was deep sequenced and found to contain no polymorphisms at greater than $5 \%$ of reads relative to the original patient isolate. The furin cleavage site, a site with frequent culture adaptation in Vero E6 cells, harboured no polymorphisms at greater than $1 \%$ of sequence reads in this stock.

\section{Sampling of nares and pharynges}

The monkeys were anaesthetized and placed in dorsal recumbency or a chair designed to maintain an upright posture. The pharynx was visualized using a laryngoscope. A sterile swab was gently rubbed and rolled across the lateral surfaces of the pharynx for approximately $5 \mathrm{~s}$, including the tonsillar fossa and posterior pharynx. Care was taken to avoid touching the soft palate, uvula, buccal mucosa, tongue or lips. After all pertinent surfaces had been sampled, the swab was removed and placed into either culture medium or an appropriate container for transport. The pharyngeal swabs were done before the nasal swabs to reduce blood contamination from the nasal cavity into the pharyngeal area.

Sterile swabs were gently inserted into the nares. Once inserted, the sponge or swab was rotated several times within the cavity or region and immediately withdrawn.

\section{BAL collection and processing}

The animals were anaesthetized using Telazol and placed in a chair designed specifically for the proper positioning for BAL procedures. A local anaesthetic ( $2 \%$ lidocaine) may be applied to the larynx at the discretion of the veterinarian. A laryngoscope was used to visualize the epiglottis and larynx. A feeding tube was carefully introduced into the trachea after which the stylet was removed. The tube was advanced further into the trachea until slight resistance was encountered. The tube was slightly retracted and the syringe attached. Aliquots of warmed normal saline were instilled into the bronchus. The saline was aspirated between each lavage before a new aliquot was instilled. When the procedure was complete, the monkey was placed in right lateral recumbency. The monkey was carefully monitored, with observation of the heart rate, respiratory rate and effort, and mucous membrane colour. An oxygen facemask may be used following the procedure at the discretion of the veterinarian. The monkey was returned to its cage, positioned on the cage floor in right lateral recumbency and was monitored closely until recovery is complete.

The BAL samples were filtered twice via $100-\mu l$ strainers and collected in 50-ml centrifuge tubes. The samples were centrifuged at $300 \mathrm{~g}$ for $10 \mathrm{~min}$ at $4{ }^{\circ} \mathrm{C}$. The supernatant was transferred into new tubes, aliquoted and stored at $-80^{\circ} \mathrm{C}$ until RNA isolation. The cells were washed, lysed for red-blood cells using ammonium-chloride-potassium (ACK) lysis buffer and live-frozen in $90 \%$ FBS with $10 \%$ DMSO.

\section{Viral load}

Quantitative PCR with reverse transcription (RT-qPCR) was performed as described previously ${ }^{41}$. RT-qPCR for the subgenomic (sg) RNA encoding the envelope (E) protein was performed as described ${ }^{42}$ and RT-qPCR for the sgRNA encoding the nucleocapsid $(\mathrm{N})$ protein was performed using the same cycling conditions as used for the $E$ sgRNA using an unpublished assay provided by D. Hartigan-O'Connor and J. Dutra (U. California-Davis). Primers and probes for the N sqRNA qRT-PCR were as follows: forward 5'-CGATCTCTTGTAGATCTGTTCTC-3', reverse 5'-GGTGAACCAAGACGCAGTAT-3', probe 5'-FAM-TAACCAGAATG
GAGAACGCAGTGGG-BHQ1-3'. Both PCRs were run in a $20 \mu \mathrm{l}$ volume containing $5 \mu$ l sample, $900 \mathrm{nM}$ primers, $250 \mathrm{nM}$ probe with TaqPath 1-step RT-qPCR master mix, CG (Thermo Fisher Scientific). The PCR conditions were $2 \mathrm{~min}$ at $25^{\circ} \mathrm{C}$ for uracil $\mathrm{N}$-glycosylase incubation, 15 min at $50^{\circ} \mathrm{C}$ for reverse transcription, 2 min at $95^{\circ} \mathrm{C}$ (Taq activation), followed by 40 cycles of $95^{\circ} \mathrm{C}$ for $3 \mathrm{~s}$ (denaturation) and $60^{\circ} \mathrm{C}$ for $30 \mathrm{~s}$ (annealing and elongation).

\section{PET-CT administration, acquisition and data collection}

The animals were anaesthetized and brought to the PET-CT suite where they were monitored and prepared for imaging. An intravenous catheter is placed and the animals were intubated and placed on a gas anaesthetic (isoflurane). FDG was administered as an intravenous bolus at a dose of $0.5 \mathrm{mCi} \mathrm{kg}^{-1}$ in the animal preparatory room. The catheter was flushed, and the animals were transferred to the PET-CT imaging room. Images were acquired on a Mediso LFER 150 PET-CT (Mediso Medical Imaging Systems). The animals were then placed on the table in a 'head-in-supine' position with heat support. Scout CT images of side and top views were obtained for positioning purposes and preferred scanning ranges. The number of fields of view (FOV) was determined depending on the size of the animal (each FOV covers $15 \mathrm{~cm}$ and takes $10 \mathrm{~min}$ to obtain with PET). ACT scan was captured at $80 \mathrm{kVp}$ and $1 \mathrm{~mA}$ with a time range of $1-5 \mathrm{~min}$ depending on the FOV. Breath holds were performed during the CT scan on animals that can be imaged in one FOV. A breath hold lasts for the majority of the CT scan which is approximately $45-60 \mathrm{~s}$. PET images were obtained following FDG uptake time (45-60 min) and the CT scan. Once the images were captured, the animal's fluids were discontinued and the animal was removed from isoflurane. When swallowing reflexes returned, the animal was extubated and returned to its home cage. Images were reconstructed using Nucline software with the following parameters: Mediso Tera-Tomo3D algorithm, 8 iterations, 9 subsets, voxel size $0.7 \mathrm{~mm}$.

\section{PET-CT data analysis}

PET-CT images were analysed using OsiriX MD or 64-bit (v.11, Pixmeo). Before analysis, the PET images were Gaussian smoothed in OsiriX and smoothing was applied to raw data with a $3 \times 3$ matrix size and a matrix normalization value of 24 . Whole lung FDG uptake was measured by first creating a whole lung region-of-interest (ROI) on the lung in the CT scan by creating a 3D growing region highlighting every voxel in the lungs between -1024 and -500 Hounsfield units. This whole lung ROI is copied and pasted to the PET scan and gaps within the ROI are filled in using a closing ROI brush tool with a structuring element radius of 4 . All voxels within the lung ROI with a standard uptake value (SUV) below 1.5 are set to zero and the SUVs of the remaining voxels are summed for a total lung FDG uptake (total inflammation) value. Total FDG uptake values were normalized to back muscle FDG uptake that was measured by drawing cylinder ROIs on the back muscles adjacent to the spine at the same axial level as the carina (SUVCMR; cylinder-muscle-ratio) ${ }^{43}$. PET quantification values were organized in Microsoft Excel. 3D images were created using the 3D volume rendering tool on OsiriX MD.

\section{Luminex isotype and FCR binding assay}

To determine relative concentrations of antigen-specific antibody isotypes and $\mathrm{Fc}$ receptor binding activity, a Luminex isotype assay was performed as previously described ${ }^{44}$. Antigens (SARS-CoV-2 spike, RBD, S1, S2, HKU1 RBD and OC43 RBD) were covalently coupled to Luminex microplex carboxylated bead regions (Luminex Corporation) using NHS-ester linkages with Sulfo-NHS and EDC (Thermo Fisher Scientific) according to manufacturer recommendations. Immune complexes were formed by incubating antigen-coupled beads with diluted samples. Mouse-anti-rhesus antibody detectors were then added for each antibody isotype (IgG1, IgG2, IgG3, IgG4 and IgA (NIH Nonhuman Primate Reagent Resource supported by Al126683 and OD010976)). 
Tertiary anti-mouse-IgG detector antibodies conjugated to phycoerythrin (PE) were then added. FcR binding was quantified similarly by using recombinant non-human primate FcRs (Fc $\gamma$ R2A-1, Fc $\gamma R 2 A-2$ and FcyR3A (Duke Protein Production Facility)) conjugated to PE as secondary detectors. Flow cytometry was performed using an iQue (Intellicyt) and an S-LAB robot (PAA), and analysis was performed on IntelliCyt ForeCyt (v 8.1).

\section{Systems serology}

To quantify antibody functionality of plasma samples, bead-based assays were used to measure antibody-dependent cellular phagocytosis (ADCP), ADNP and antibody-dependent complement deposition (ADCD), as previously described ${ }^{45-48}$. SARS-CoV-2 spike protein (HexaPro antigen (from E. Ollmann Saphire, La Jolla Institute for Immunology)) was coupled to fluorescent streptavidin beads (Thermo Fisher) and incubated with serum samples to allow antibody binding to occur. For ADCP, cultured human monocytes (THP-1 cell line) were incubated with immune complexes, during which phagocytosis occurred. For ADNP, primary PMBCs were isolated from whole blood using an ACK lysis buffer. After phagocytosis of immune complexes, neutrophils were stained with an anti-CD66b Pacific Blue detection antibody (Biolegend) before flow cytometry. For ADCD, lyophilized guinea pig complement (Cedarlane) was reconstituted according to manufacturer's instructions and diluted in a gelatin veronal buffer with calcium and magnesium (Boston BioProducts). After antibody-dependent complement deposition occurred, $\mathrm{C} 3$ bound to immune complexes was detected with FITC-Conjugated Goat IgG Fraction to Guinea Pig Complement C3 (MP Biomedicals). For quantification of antibody-dependent natural killer (NK) cell activation, diluted plasma samples were incubated in Nunc MaxiSorp plates (Thermo Fisher Scientific) coated with antigen. Human NK cells were isolated the evening before using RosetteSep Human NK cell Enrichment cocktail (Stemcell Technologies) from healthy buffy coat donors and incubated overnight with human recombinant IL-15 (STEMCELL Technologies). NK cells were incubated with immune complexes, CD107a PE-Cy5 (BD), Golgi stop (BD) and brefeldin A (Sigma-Aldrich). After incubation, cells were stained using anti-CD16 APC-Cy7 (BD), anti-CD56 PE-Cy7 (BD) and anti-CD3 Pacific Blue (BD), and then fixed (Perm A, Life Tech). Intracellular staining using anti-IFN $\gamma$ FITC (BD) and anti-MIP-1 $\beta$ PE (BD) was performed after permeabilizing the NK cells with Perm $B$ (Thermo Fisher). Flow cytometry acquisition of all assays was performed using an iQue (IntelliCyt) and a S-LAB robot (PAA). For ADCP, phagocytosis events were gated on bead-positive cells. For ADNP, neutrophils were identified by gating on $\mathrm{CD}_{6} 6 \mathrm{~b}^{+}$cells, phagocytosis was identified by gating on bead-positive cells. A phagocytosis score for ADCP and ADNP was calculated as (percentage of bead-positive cells) $\times$ (MFI of bead-positive cells) divided by 10,000. ADCD quantification was reported as MFI of FITC-anti-C3. For antibody-dependent NK activation, NK cells were identified by gating on $\mathrm{CD}^{-}, \mathrm{CD}^{+} 6^{+}$and $\mathrm{CD} 56^{+}$cells. Data were reported as the percentage of cells positive for CD107a, IFN $\gamma$ and MIP-1 $\beta$.

\section{Statistics and data visualization}

The difference between any two groups at a time point was measured using a two-tailed nonparametric Mann-Whitney unpaired rank-sum test. The difference between time points within a group was measured using a Wilcoxon matched-pairs signed-rank test. All correlations were Spearman's correlations based on ranks. All the statistical analyses were performed using GraphPad Prism v.9.0.0 or R version 3.6.1. All the figures were made in GraphPad Prism or R and organized in Adobe Illustrator.

\section{Reporting summary}

Further information on research design is available in the Nature Research Reporting Summary linked to this paper.

\section{Data availability}

All data from the study are included in the manuscript and associated files. Source data are provided with this paper.

32. Tiller, T. et al. Efficient generation of monoclonal antibodies from single human B cells by single cell RT-PCR and expression vector cloning. J. Immunol. Methods 329, 112-124 (2008).

33. Millet, J. K. \& Whittaker, G. R. Murine leukemia virus (MLV)-based coronavirus spike-pseudotyped particle production and infection. Bio Protoc. 6, e2035 (2016).

34. Pinto, D. et al. Cross-neutralization of SARS-CoV-2 by a human monoclonal SARS-CoV antibody. Nature 583, 290-295 (2020).

35. Crawford, K. H. D. et al. Protocol and reagents for pseudotyping lentiviral particles with SARS-CoV-2 spike protein for neutralization assays. Viruses 12, E513 (2020).

36. Vanderheiden, A. et al. Development of a rapid focus reduction neutralization test assay for measuring SARS-CoV-2 neutralizing antibodies. Curr. Protoc. Immunol. 131, e116 (2020).

37. Xie, X. et al. An infectious cDNA clone of SARS-CoV-2. Cell Host Microbe 27, 841-848 (2020).

38. Katzelnick, L. C. et al. Viridot: an automated virus plaque (immunofocus) counter for the measurement of serological neutralizing responses with application to dengue virus. PLoS Negl. Trop. Dis. 12, e0006862 (2018).

39. Weissman, D. et al. D614G spike mutation increases SARS CoV-2 susceptibility to neutralization. Cell Host Microbe 29, 23-31 (2021).

40. Tegally, $\mathrm{H}$. et al. Emergence and rapid spread of a new severe acute respiratory syndrome-related coronavirus 2 (SARS-CoV-2) lineage with multiple spike mutations in South Africa. Preprint at https://doi.org/10.1101/2020.12.21.20248640 (2020).

41. Blair, R. V. et al. Acute respiratory distress in aged, SARS-CoV-2-infected African green monkeys but not rhesus macaques. Am. J. Pathol. 191, 274-282 (2021).

42. Wölfel, R. et al. Virological assessment of hospitalized patients with COVID-2019. Nature 581, 465-469 (2020).

43. White, A. G. et al. Analysis of 18FDG PET/CT imaging as a tool for studying Mycobacterium tuberculosis infection and treatment in non-human primates. J. Vis. Exp. 127, 56375

44. Brown, E. P. et al. High-throughput, multiplexed IgG subclassing of antigen-specific antibodies from clinical samples. J. Immunol. Methods 386, 117-123 (2012).

45. Ackerman, M. E. et al. A robust, high-throughput assay to determine the phagocytic activity of clinical antibody samples. J. Immunol. Methods 366, 8-19 (2011).

46. Fischinger, S. et al. A high-throughput, bead-based, antigen-specific assay to assess the ability of antibodies to induce complement activation. J. Immunol. Methods 473, 112630 (2019).

47. Karsten, C. B. et al. A versatile high-throughput assay to characterize antibody-mediated neutrophil phagocytosis. J. Immunol. Methods 471, 46-56 (2019).

48. Lu, L. L. et al. A functional role for antibodies in tuberculosis. Cell 167, 433-443 (2016).

Acknowledgements This study was supported by the Bill and Melinda Gates Foundation INV018675 to B.P. and INV-017592 to J.S.M.; OPP1156262 to D.V. and N.P.K., a generous gift from the Audacious Project, a generous gift from J. Green and M. Halperin, a generous gift from the Hanauer family, the Defense Threat Reduction Agency (HDTRA1-18-1-0001 to N.P.K.), the National Institute of General Medical Sciences (R01GM120553 to D.V.), the National Institute of Allergy and Infectious Diseases (DP1Al158186 and HHSN272201700059C to D.V.; and R01-Al127521 to J.S.M.), a Pew Biomedical Scholars Award (D.V.), Investigators in the Pathogenesis of Infectious Disease Awards from the Burroughs Wellcome Fund (D.V.), Fast Grants (D.V.); Bill and Melinda Gates Foundation INV - 018417 (J.L.F.); and NIAID-NIH Contract: HHSN272201800004C to D.M. We thank the staff at the New Iberia Research Center, UL Lafayette and the Tulane National Primate Research Center for conducting the animal studies; M. Pepper for coordination of human convalescent samples; and L. Stuart at the Bill and Melinda Gates Foundation for inputs and insights throughout the study; all the members of GSK, Dynavax and Seppic for critical reading of the manuscript. We acknowledge the generous help of Rashmi Ravichandran for protein production. The schematics were made using BioRender.

Author contributions B.P., N.P.K. and H.K. conceptualized the study; B.P., P.S.A., A.C.W., F.V. N.P.K., D.V. and J.R. designed the study and were responsible for overall conduct of the study; S.W., D.P., M.C.M., E.K., C.S., N.B., M.M., B.F. and L.C. produced and purified RBD-NP and HexaPro-NP immunogens under the supervision of N.P.K.; J.F. and K. Rogers organized and performed all macaque immunizations under the supervision of F.V.; L.S., D.E.F. and K. Rogers processed all samples collected during the immunization phase; P.S.A. and K. Röltgen performed binding ELISA in Fig. 1 under the supervision of S.D.B.; A.C.W. and M.J.N, performed pseudovirus neutralizing-antibody response assays against the wild-type virus under the supervision of D.V.; V.V.E. and L.L. performed all authentic virus neutralizing-antibody assays under the supervision of M.S.S.; J.C.K. performed anti-spike and anti-NP binding-antibody assays; P.S.A., C.L. and M.T. performed T cell assays; N.G. and P.A., organized all challenge experiments. N.G., P.A., K.R.-L., J.D., L.D.-M., C.M., R.P.B. and N.J.M. performed all post-challenge experiments; J.A.P., K.S.P. and C.R. provided challenge virus; A.G.W. and J.L.F. analysed PET-CT data; P.S.A. and S.G. performed immune correlates analysis under the supervision of S. Subramaniam; C.A., S.F., A.Z., M.J.G. and S. Shin performed, analysed and prepared figures of systems serology under the supervision of G.A.; C.-L.H. and J.S.M. provided HexaPro; X.S. and D.M. measured pseudovirus neutralizing-antibody response against the B.1.1.7 variant; D.T.O., R.V.D.M. and R.R. provided $\mathrm{ASO} 3$ and $\mathrm{AS} 37$ and guided formulation with the two adjuvants; R.L.C. and D.N. provided and guided CpG 1018 and its formulation with alum; H.K. provided guidance throughout the project. P.S.A. and B.P. were responsible for the formal analysis of all datasets and preparation of figures; P.S.A. and B.P. wrote the manuscript with suggestions and assistance from all co-authors. All the authors read and accepted the final contents of the manuscript. 
Competing interests B.P. serves on the External Immunology Board of GlaxoSmithKline, and on the Scientific Advisory Board of Medicago. D.T.O., R.V.D.M. and R.R. are employees of the GSK group of companies. R.L.C. and D.N. are employees of Dynavax Technologies Corporation. H.K. is an employee of Bill and Melinda Gates Foundation. C.-L.H. and J.S.M. are inventors on US patent application no. 63/032,502 'Engineered Coronavirus Spike (S) Protein and Methods of Use Thereof'. N.P.K. is a co-founder, shareholder, paid consultant, and chair of the scientific advisory board of Icosavax, Inc. and has received an unrelated sponsored research agreement from Pfizer. D.V. is a consultant for Vir Biotechnology Inc. The Veesler laboratory has received an unrelated sponsored research agreement from Vir Biotechnology Inc.
Additional information

Supplementary information The online version contains supplementary material available at https://doi.org/10.1038/s41586-021-03530-2.

Correspondence and requests for materials should be addressed to B.P.

Peer review information Nature thanks Alan Barrett, Wolfgang Baumgärtner and the other, anonymous, reviewer(s) for their contribution to the peer review of this work. Peer reviewer reports are available.

Reprints and permissions information is available at http://www.nature.com/reprints. 


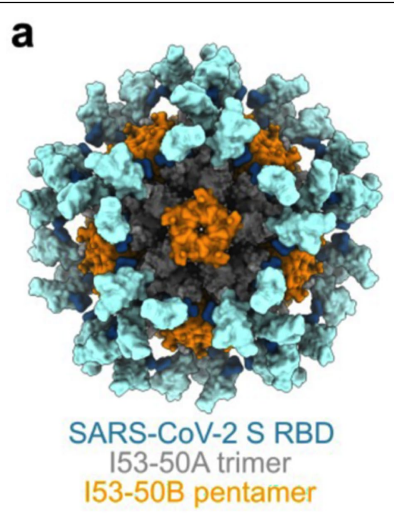

153-50B pentamer
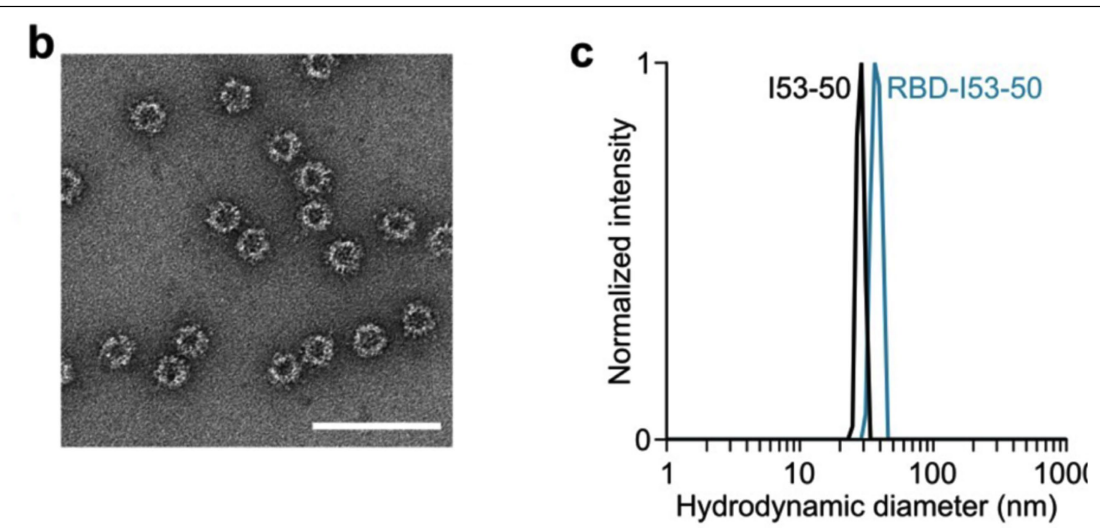
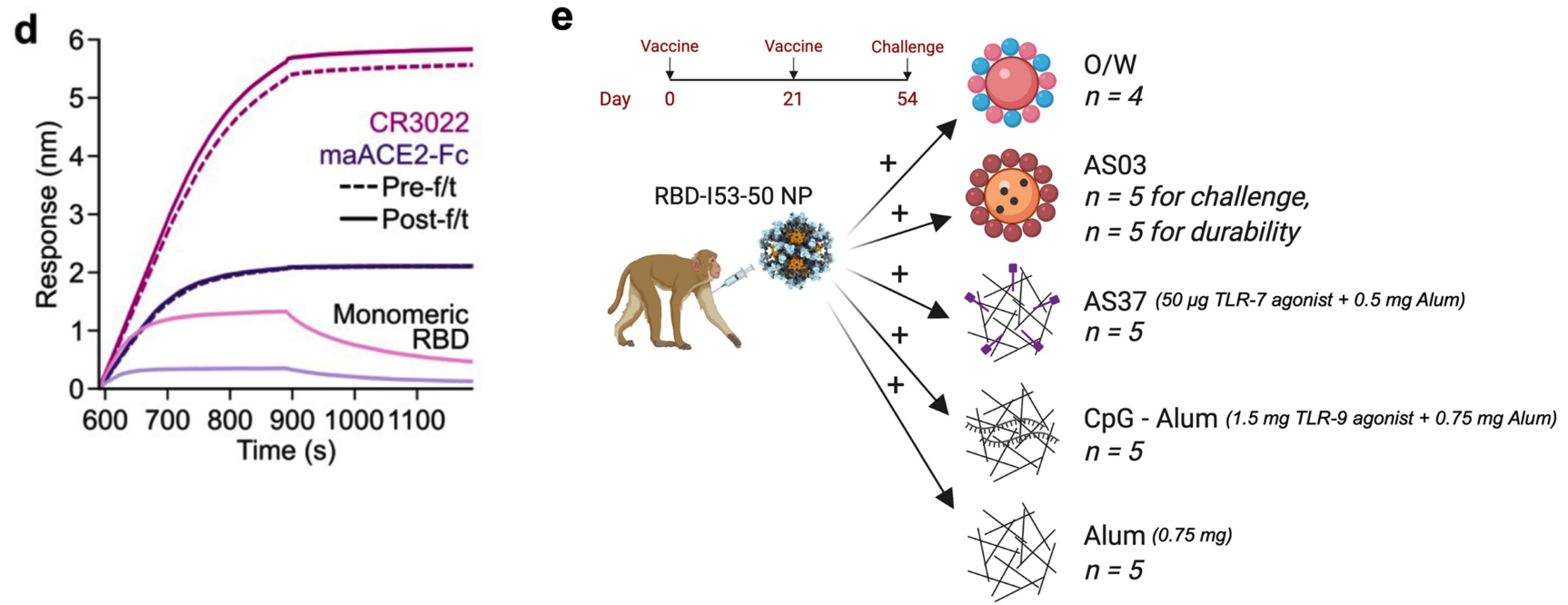

\section{f Anti-Spike and anti-NP IgG responses}

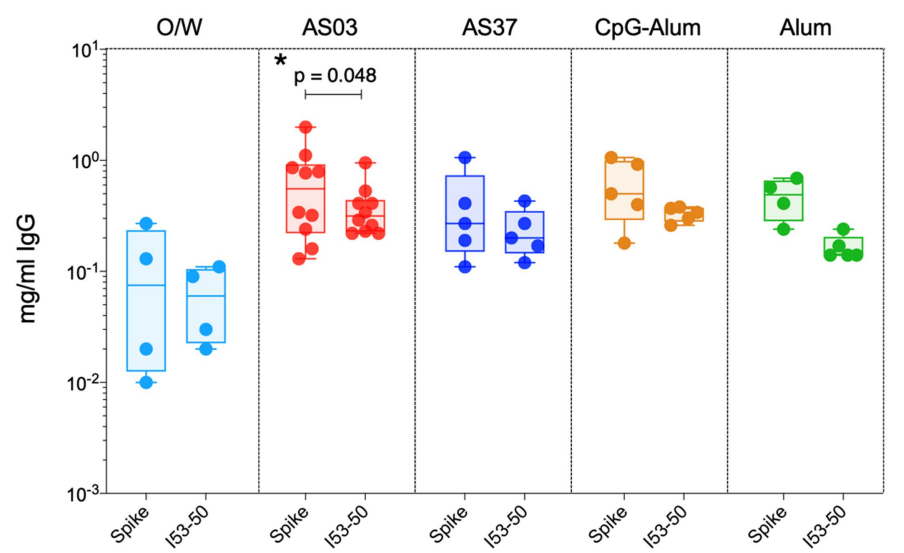

\section{g NP-vs. Spike-specific lgG responses}

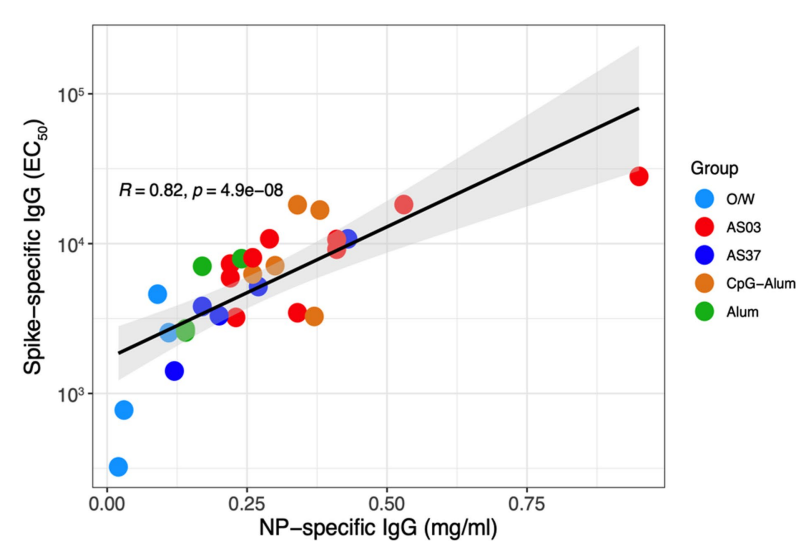

thaw cycle. Monomeric SARS-CoV-2 RBD was used as a reference antigen. e, Schematic representation of the study design. $\mathbf{f}$, Serum concentrations of anti-spike IgG and anti-I53-50 NP IgG (anti-I53-50) in individual non-human primates detected by ELISA at day 42. Boxes show median, 25th and 75 th percentiles and the whiskers show the range. The statistical difference between anti-spike and anti-I53-50 IgG response was determined using two-sided Wilcoxon matched-pairs signed-rank test. g, Spearman's correlation between anti-spike IgG (described in Fig. 1) and anti-NP IgG responses at day 42 . The error bands represent $95 \%$ confidence limits. Each symbol represents an animal. $N=4$ for $\mathrm{O} / \mathrm{W}, 10$ for ASO3 and 5 for all other groups. by biolayer interferometry (BLI). RBD-NP was bound to immobilized CR3022 monoclonal antibody and maACE2-Fc receptor, both before and after one freeze/ 
a Pseudovirus $\mathrm{nAb}$ titers

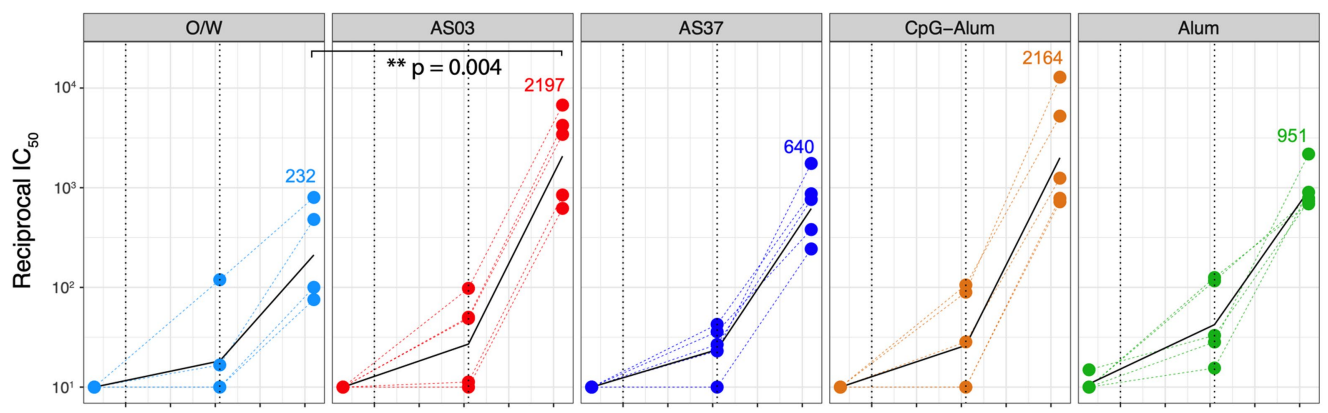

b

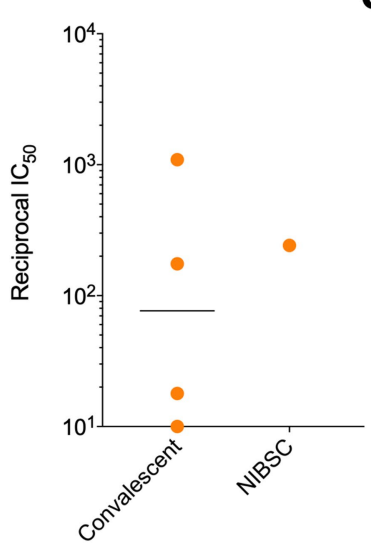

e Correlation with ARCs

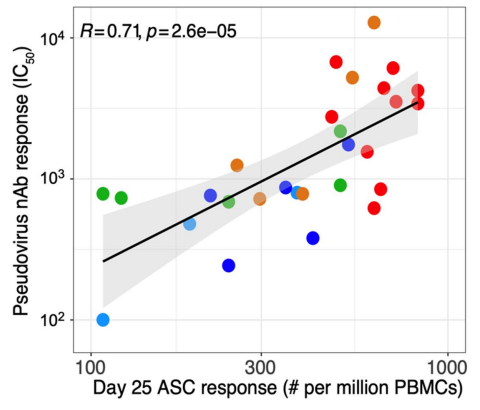

h Cross-neutralization

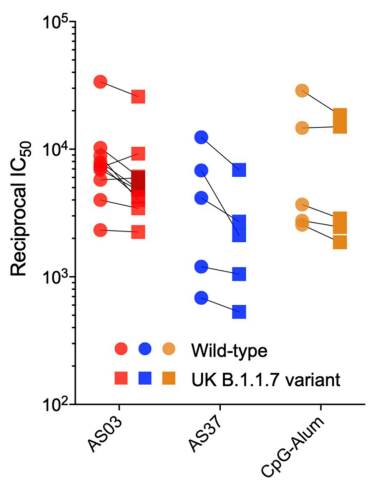

Days post immunization

C Correlation between $\mathrm{nAb}$ titers

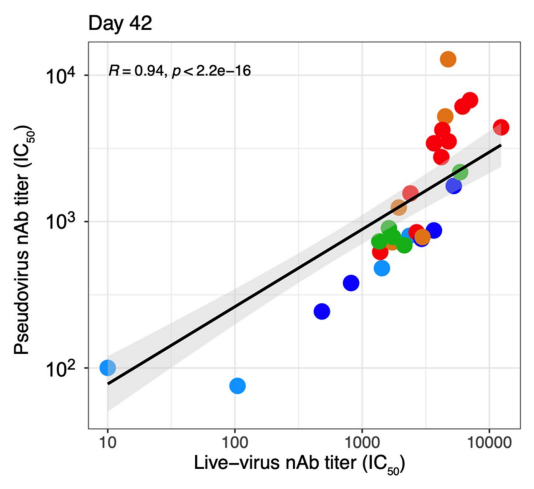

f Durability of pseudovirus $n A b$ titers

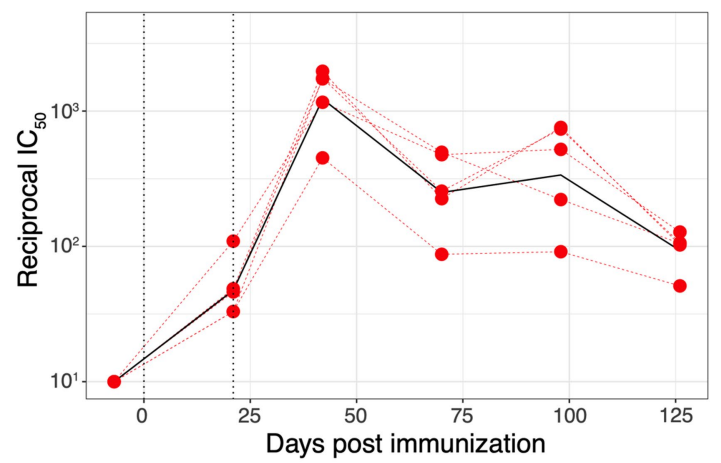

d RBD-NP-specific ASPs

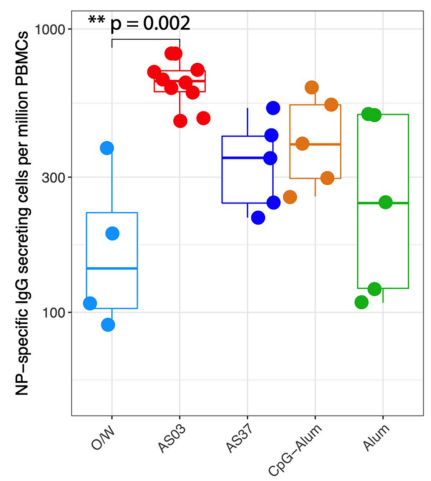

9 ACE-2 blocking

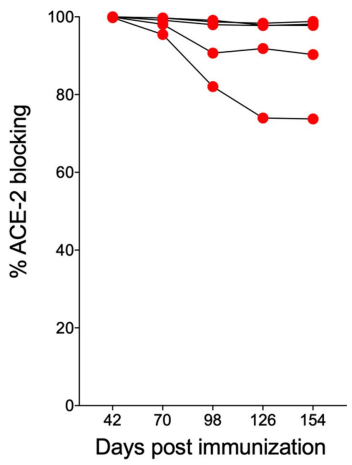

Extended Data Fig. 2 |See next page for caption. 


\section{Article}

Extended Data Fig. 2 | Antibody responses to adjuvanted RBD-NP

immunization. a, Serum neutralizing antibodies ( $\mathrm{nAb}$ ) titres determined using a SARS-CoV-2S pseudovirus entry assay at day $-7,21$ and 42 .

b, Pseudovirus $n A b$ response against human convalescent sera from 4 COVID-19 patients.c, Spearman's correlation between pseudovirus and authentic virus $n A b$ titres measured at day 42.d, RBD-NP-specific IgG secreting plasmablast response measured at day 4 post-secondary vaccination using ELISPOT. Boxes show median, 25th and 75th percentiles and the whiskers show the range.e, Spearman's correlation between plasmablast response on day 25 and pseudovirus nAb titre measured at day 42.f, Pseudovirus $n A b$ response measured in the AS03 durability group at time points indicated on the $x$ axis.g, ACE2 blocking measured in sera collected at time points indicated on the $x$ axis. h, SARS-CoV-2 nAb titres against pseudovirus wild-type containing D614G mutation on the Wuhan-1 spike (circles) or the B.1.1.7 variant (squares) strain measured in day 42 sera. The difference between groups in $\mathbf{a}$ and $\mathbf{d}$ was analysed using two-sided Mann-Whitney rank-sum test. The error bands in c and e represent $95 \%$ confidence limits. $N=4$ for $\mathrm{O} / \mathrm{W}, 10$ for $\mathrm{ASO} 3$ and 5 for all other groups. 
a RBD-specific CD4 T cell responses

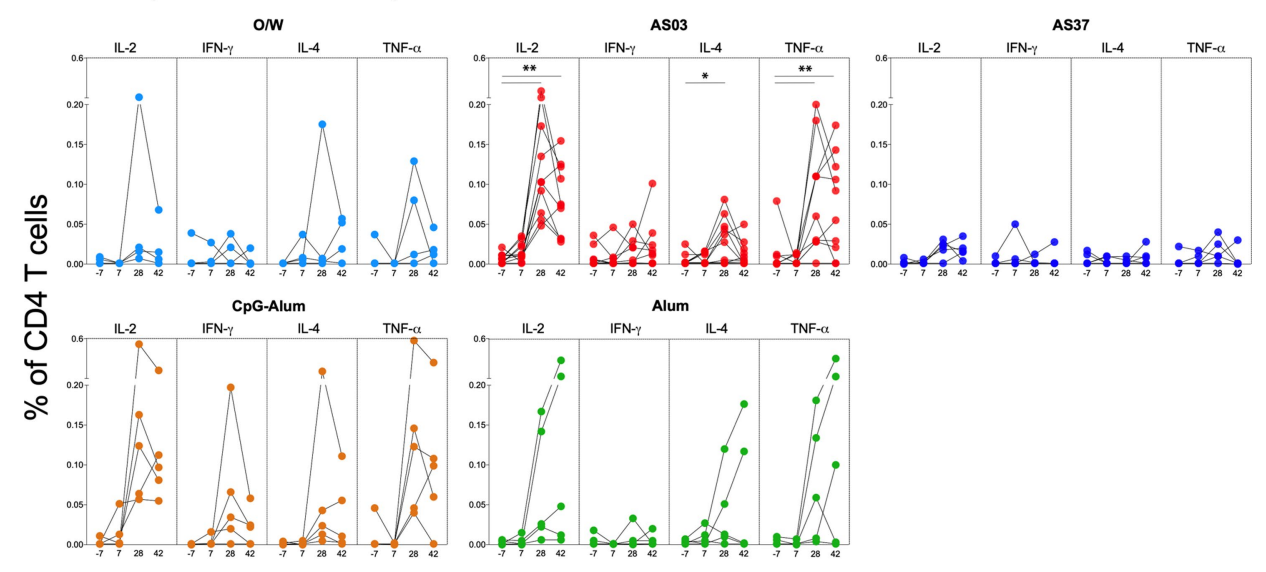

b RBD-specific polyfunctional CD4 T cells

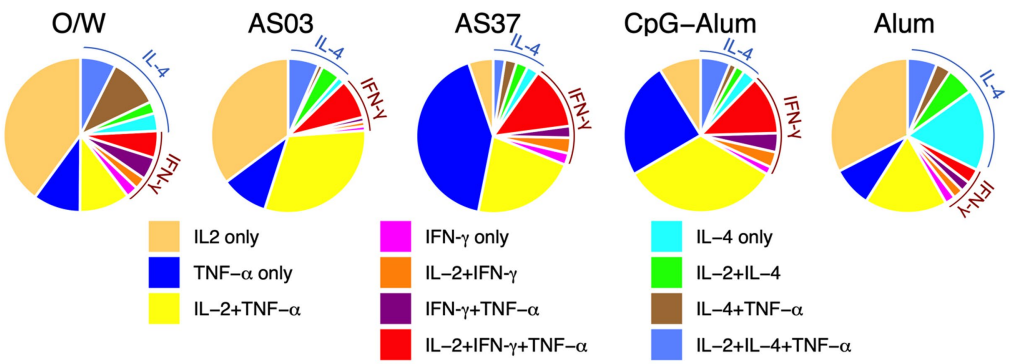

C NP-specific CD4 T cell responses

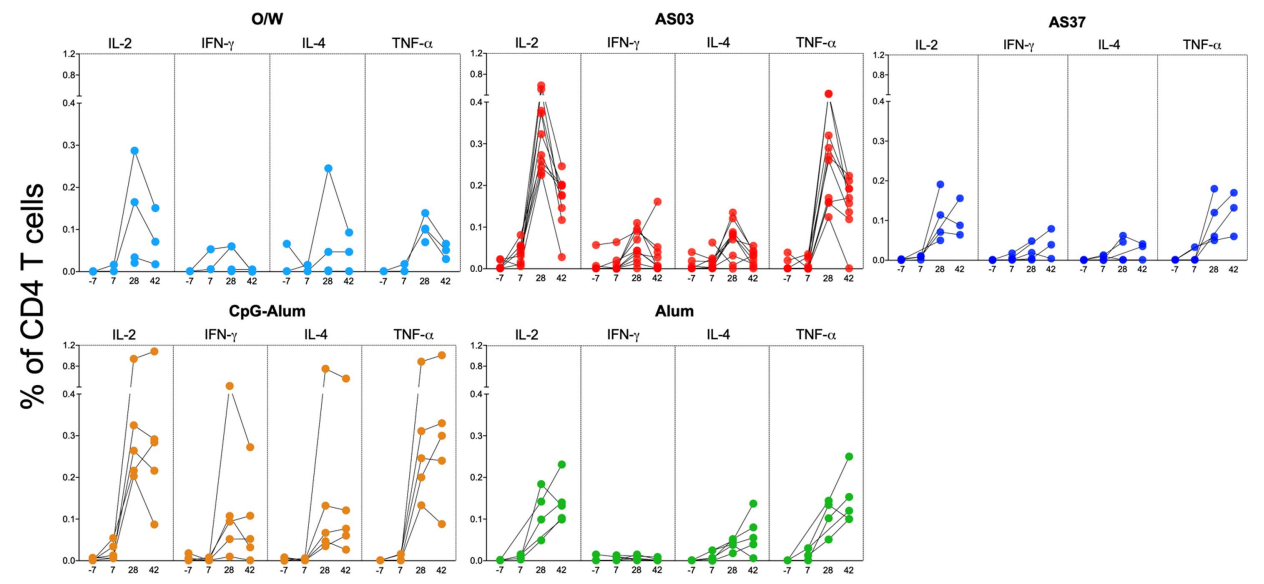

d Ratio of RBD- to NP-specific CD4 T cells

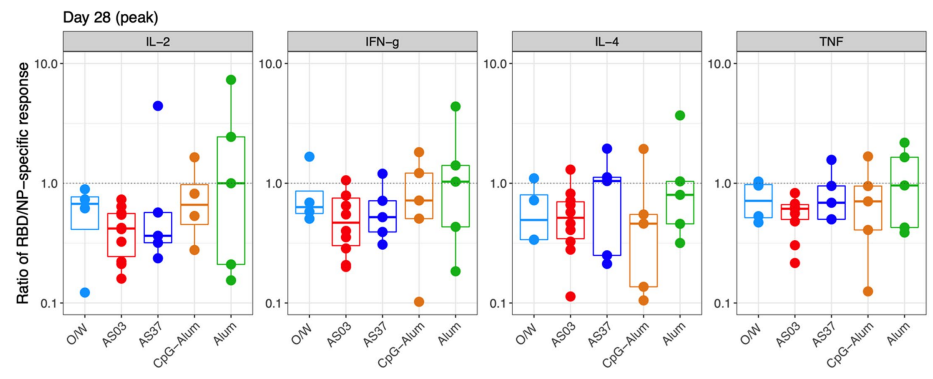

Extended Data Fig. 3 | Cell-mediated immune responses to RBD-NP immunization. a, RBD-specific CD4 T cell responses measured in blood at time points indicated on the $x$ axis. The differences between time points within a group were analysed by two-sided Wilcoxon matched-pairs signed-rank test $\left({ }^{*} P=0.016\right.$, $\left.{ }^{* *} P<0.01\right)$. b, Pie charts representing the proportions of RBD-specific CD4 T cells expressing one, two, or three cytokines as shown in the legend.c, NP-specific CD4
T cell responses in blood at time points indicated on the $x$ axis. d, Ratio of frequencies of RBD-specific to NP-specific CD4 T cells expressing cytokines indicated within each plot. Boxes show median, 25 th and 75 th percentiles and the whiskers show the range. The dotted horizontal lines indicate a ratio of $1 . N=4$ for $\mathrm{O} / \mathrm{W}, 10$ for $\mathrm{ASO} 3$ and 5 for all other groups. 
a Pharyngeal swab

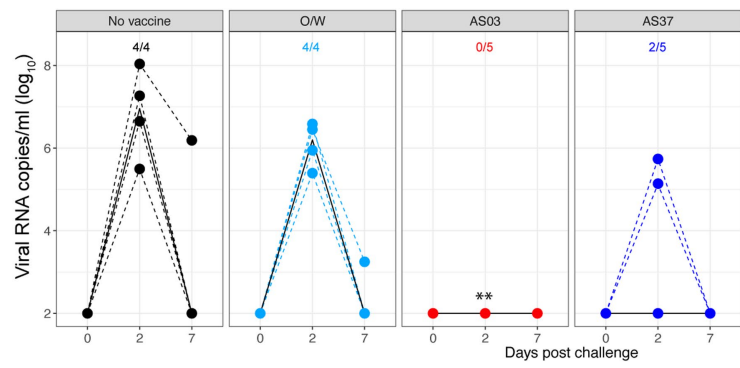

b
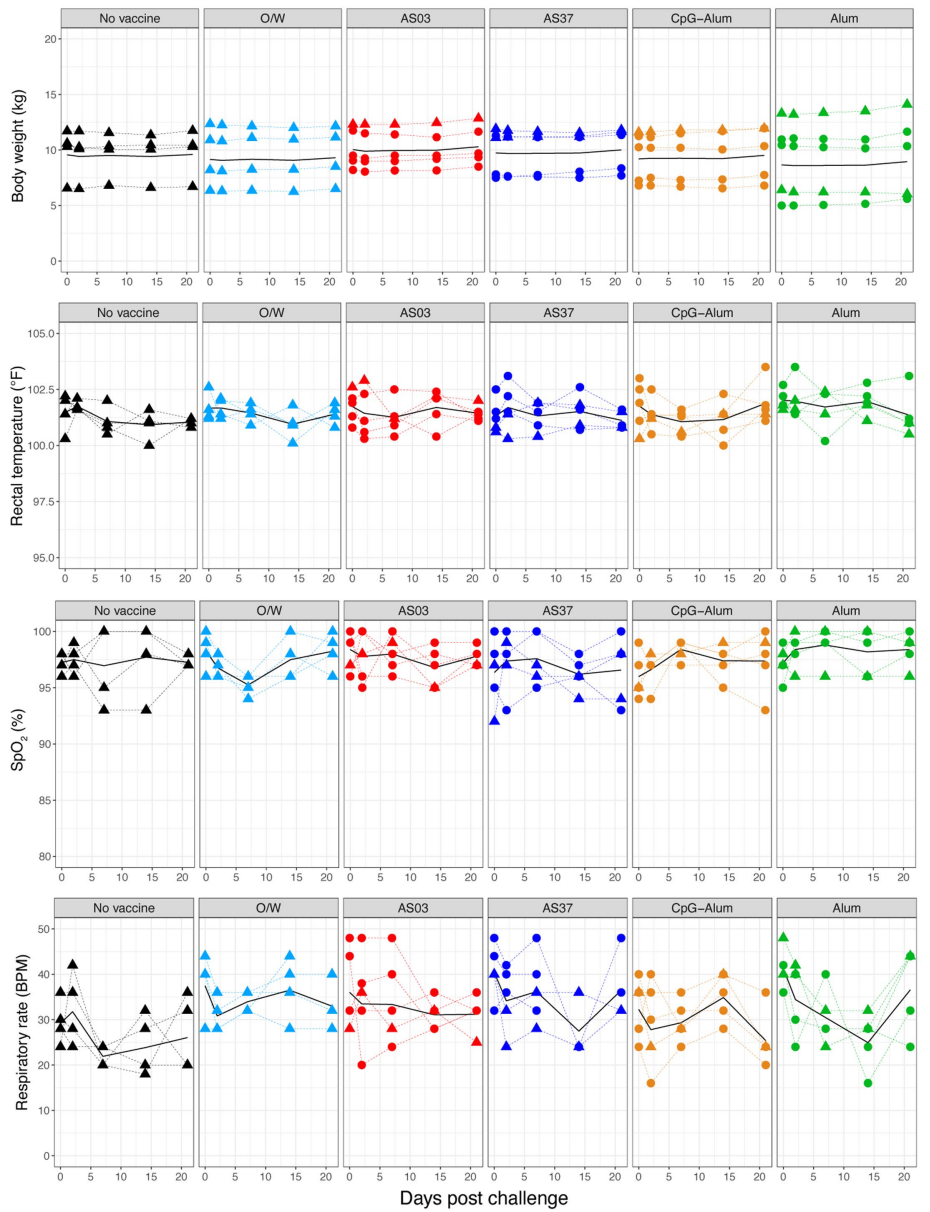

C

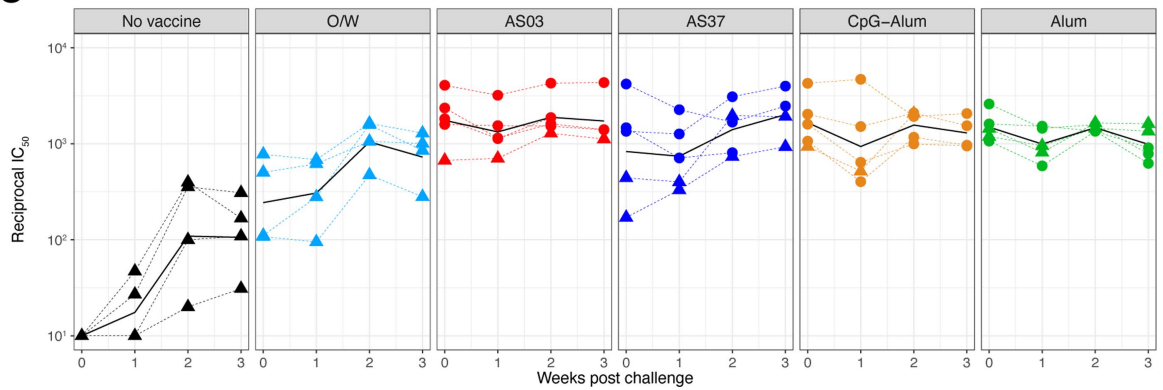

No vaccine 2

AS03 1

CpG-Alum 1

Cpg-Alum 2

AS03 2 d PET-CT

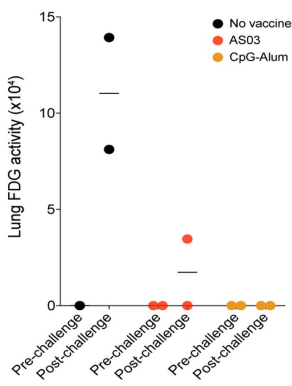

e

Pre-

Postchallenge challenge
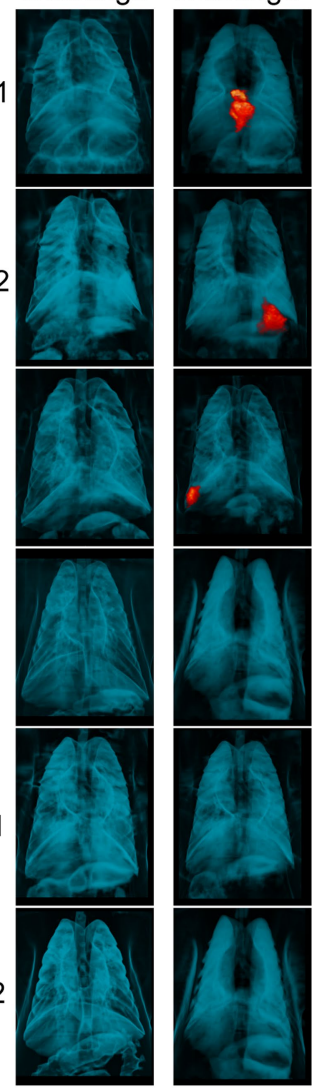

Extended Data Fig. 4 |See next page for caption. 
Extended Data Fig. 4 | Analysis of immune responses post SARS-CoV-2 challenge. a, SARS-CoV-2 viral load in pharynges measured using subgenomic PCR. The numbers within the plots denote number of infected animals per total number of animals within each group. Asterisks indicate statistically significant differences in comparison to the no vaccine control group determined using two-sided Mann-Whitney rank-sum $\left(^{* *} P=0.008\right)$. b. Clinical parameters measured on the day of challenge, 2 days, 1-, 2- and 3-weeks post SARS-CoV-2 challenge. Body weight $(\mathrm{kg})$, body temperature $\left({ }^{\circ} \mathrm{F}\right)$, Oxygen saturation $\left(\mathrm{SpO}_{2}\right)$ and respiratory rate (BPM) are shown in first, second, third and fourth rows, respectively. $c$, Serum nAb titres (plotted as reciprocal $\mathrm{IC}_{50}$ ) determined using a SARS-CoV-2 $\mathrm{S}$ pseudovirus entry assay on the day of challenge, 1,2 and 3 weeks post challenge. The black line represents the geometric mean of all data points. The circle and triangle shape of the points represent animals protected or infected (in any compartment, that is, nares, pharynges or BAL), respectively. $N=4$ for no vaccine and $\mathrm{O} / \mathrm{W}$ groups and 5 for all other groups. d, FDG activity in the lungs of two animals from each group indicated in the legend, pre-challenge (day 0 ) and post-challenge (day 4 or 5 after infection), measured using PET-CT scans. e, PET-CT images obtained from the lungs of SARS-CoV-2 infected animals from no vaccine, AS03, or CpGAlum groups pre-challenge (day 0 ) and post-challenge (day 4 or 5). PET signal is scaled 0 to 15 SUV and shown in red. Each symbol represents an animal. 


\section{Article}

a

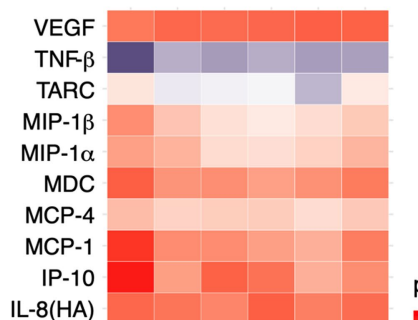

IL-8(HA)

IL-8
IL-7

IL-6

IL-5

IL-2

IL-1 $\beta$

IL-17A

IL-16

IL-15

IL-12/IL-23p40

IL-10

IFN- $\gamma$

GM-CSF

Eotaxin-3

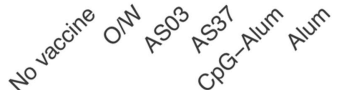

Extended Data Fig. 5 | Cytokine analysis in BAL fluid post SARS-CoV-2 challenge. a, Heat map showing expression of 24 cy tokines measured in BAL fluid collected 1 week post SARS-CoV-2 challenge. b, Expression of Eotaxin- 3 (CCL26), an eosinophil-recruiting chemokine known to be induced by the $\mathrm{T}_{\mathrm{H}} 2$ cytokine IL-13, and IL-5, a $\mathrm{T}_{\mathrm{H}} 2$ cytokine in the BAL fluid collected 1 week post challenge shows no significant increase in vaccinated animals compared to no
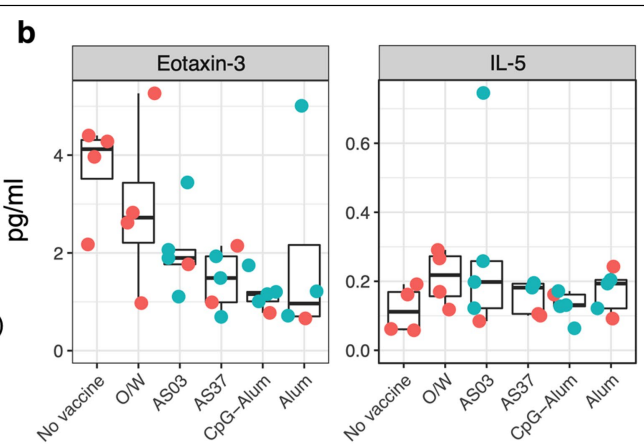

- Protected

- Infected
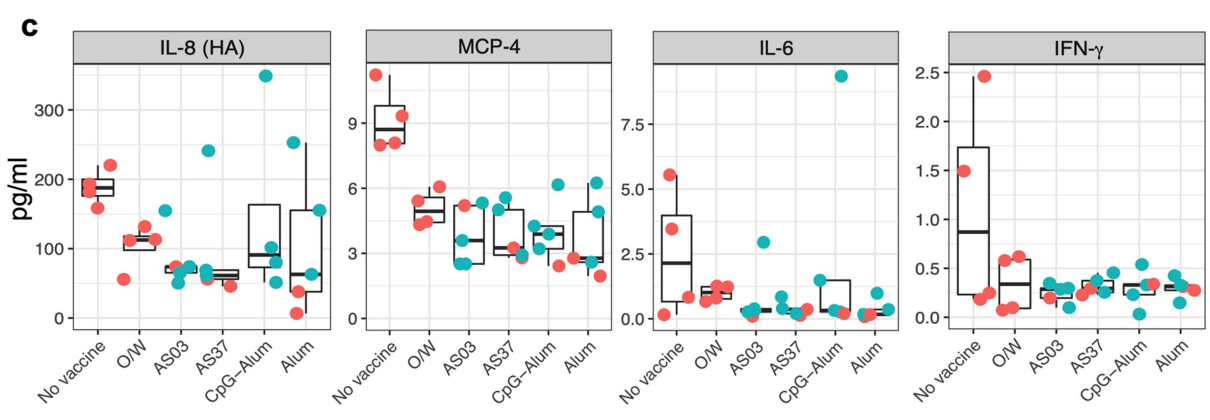

vaccine controls. c, Abundance of cytokines known to be induced by SARS-CoV-2 infection in humans such as IL-8, MCP4, IL-6 and IFN $\gamma$ in BAL collected 1 week post challenge. All the box plots show median, 25 th and 75 th percentiles and the whiskers show the range. $N=4$ for control and $\mathrm{O} / \mathrm{W}, 5$ for all othergroups. 
a

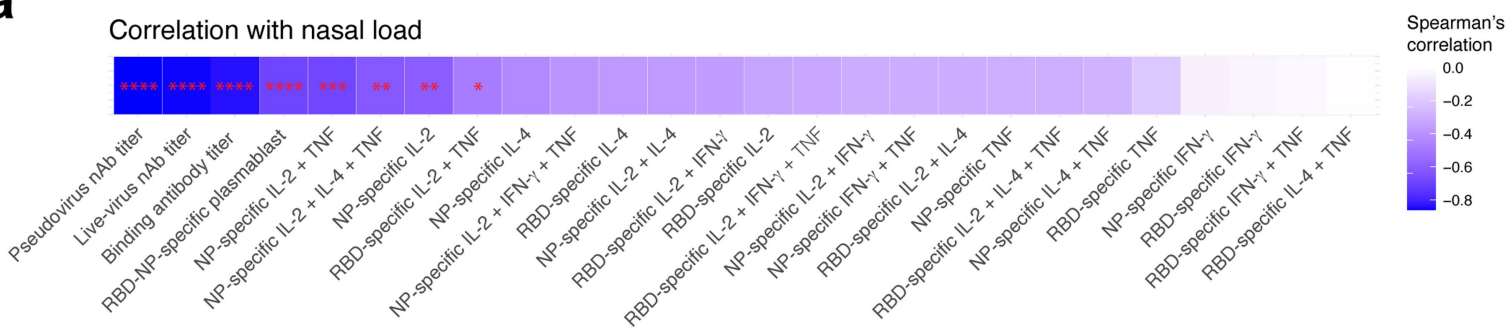

b Correlation with pharyngeal load

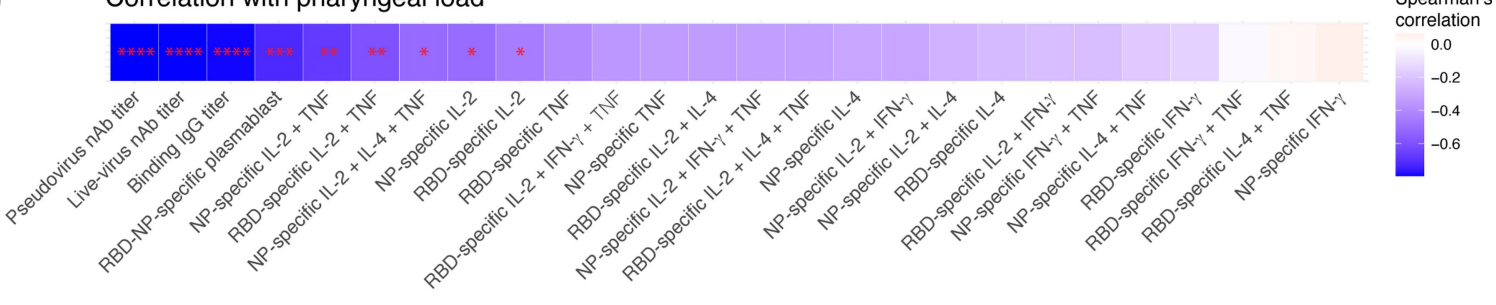

\section{T cell correlate in nasal and pharyngeal compartments}

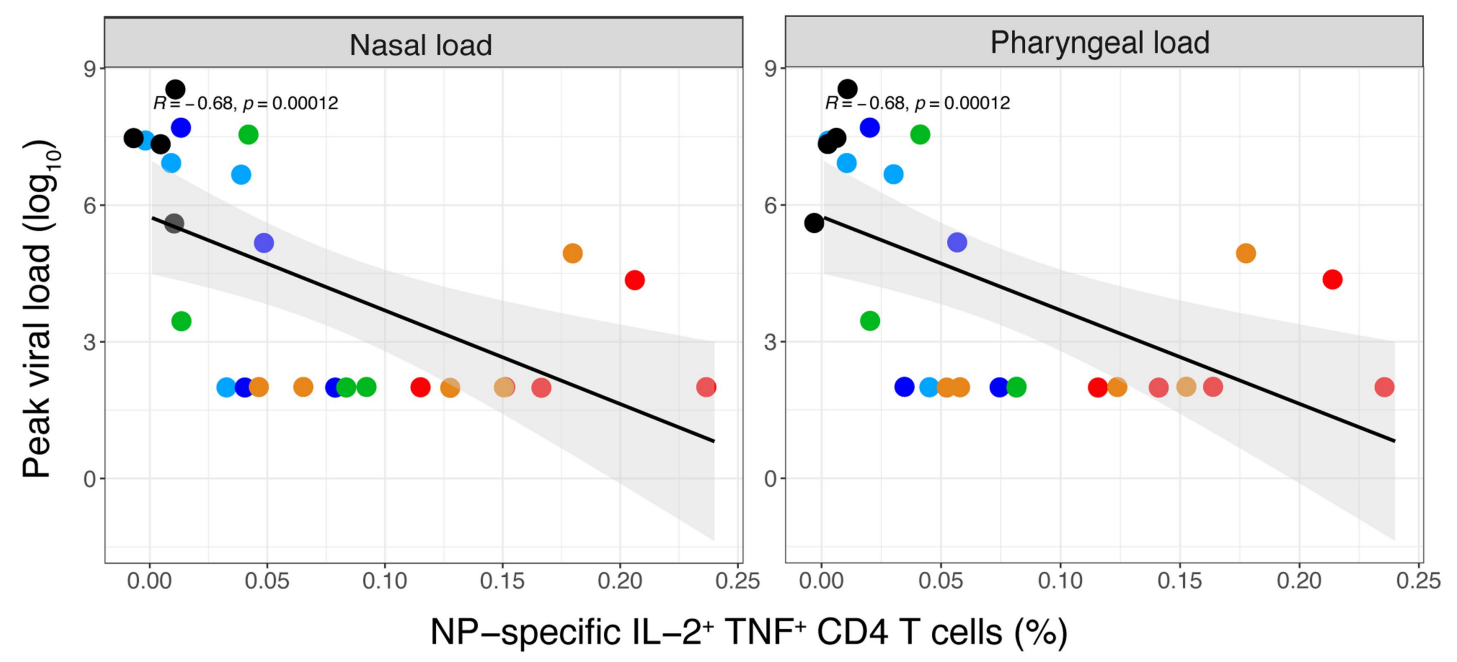

\section{d Correlation between $\mathrm{T}$ cells and nAbs}

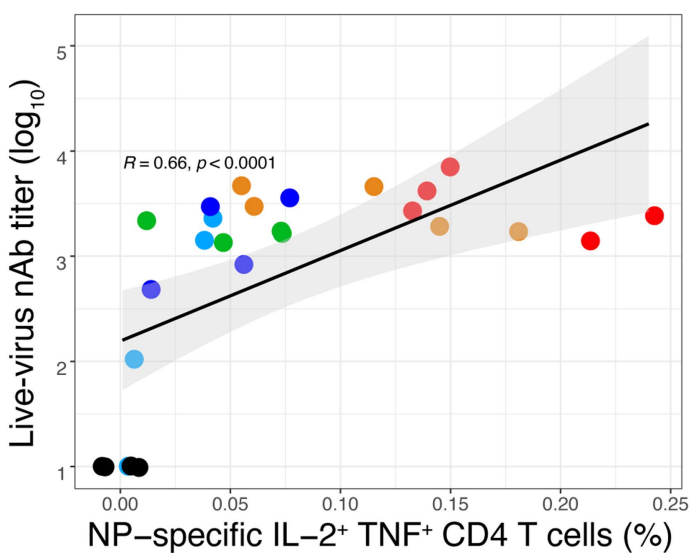

Extended Data Fig. 6 | Immune correlates of protection. a, b, Heat map showing two-sided Spearman's correlation between peak nasal (a) and pharyngeal (b) viral load (day 2) and various immune parameters. All measurements were from peak time points (day 42 for antibodies, day 25 for plasmablast, and day 28 for $\mathrm{T}$ cell responses). The $P$ values were corrected for multiple-testing. Asterisks represent statistical significance $\left({ }^{*} P<0.05,{ }^{* *} P<0.01\right.$, ${ }^{* * *} P<001$ and $\left.{ }^{* * * *} P<0.0001\right)$. c, Spearman's correlation plots between peak nasal
Group
No vaccine
O/W
AS03
AS37
CpG-Alum
Alum

(left) or pharyngeal (right) viral load and the frequency of NP-specific IL-2 ${ }^{+} \mathrm{TNF}^{+}$ CD4 $\mathrm{T}$ cells measured at day 28,1 week after secondary immunization. d, Spearman's correlation between the frequency of NP-specific IL-2 ${ }^{+} \mathrm{NNF}^{+} \mathrm{CD} 4$ $\mathrm{T}$ cells measured at day 28 and nAb response measured on day 42 . The error bands in $\mathbf{b}$ and $\mathbf{c}$ represent $95 \%$ confidence limits. $N=4$ for control and $\mathrm{O} / \mathrm{W}, 5$ for all other groups. 

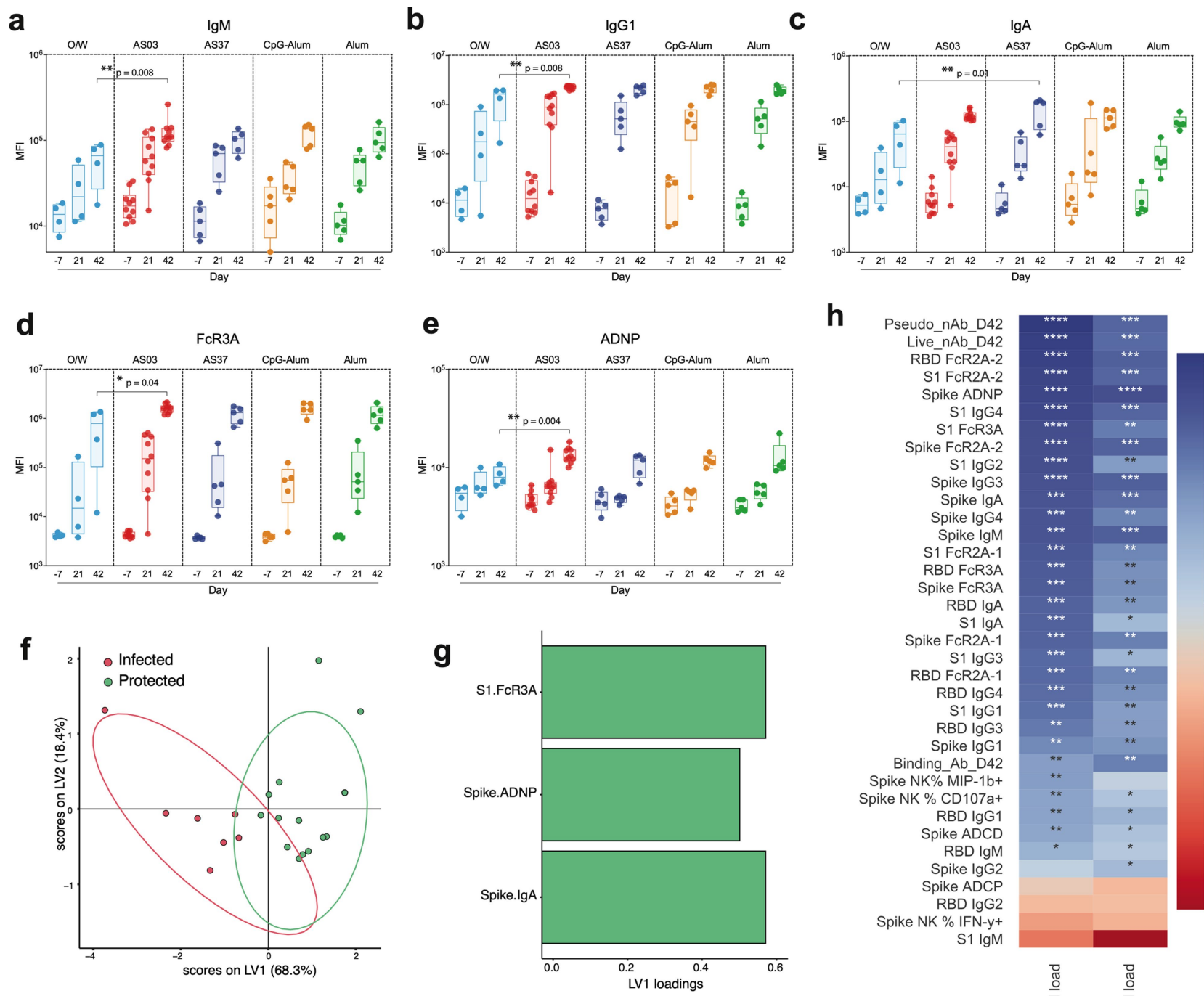

h

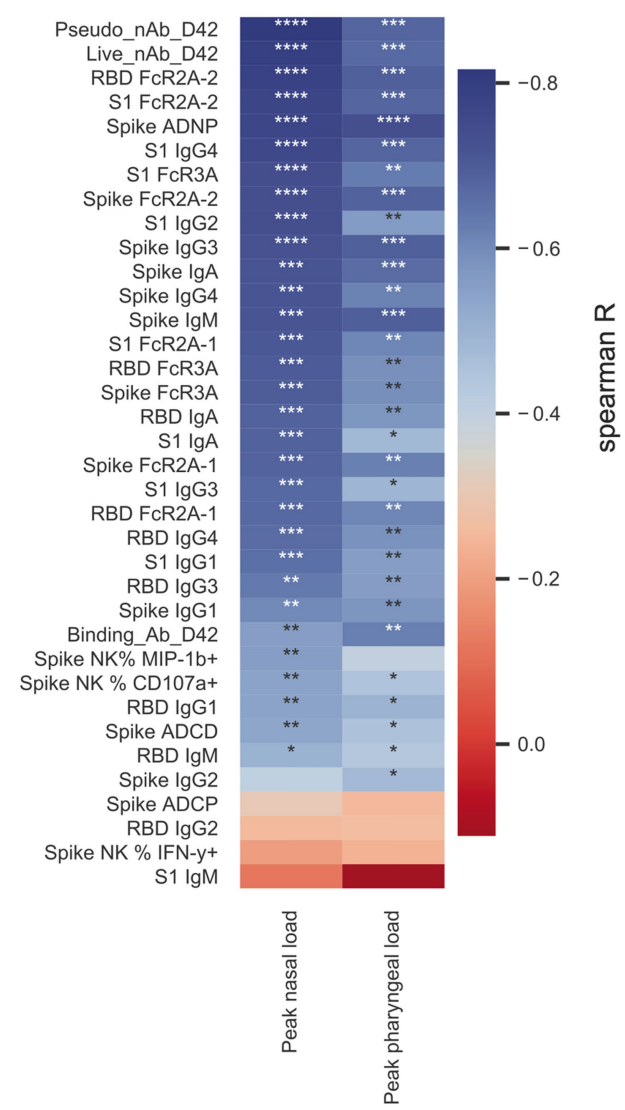

i
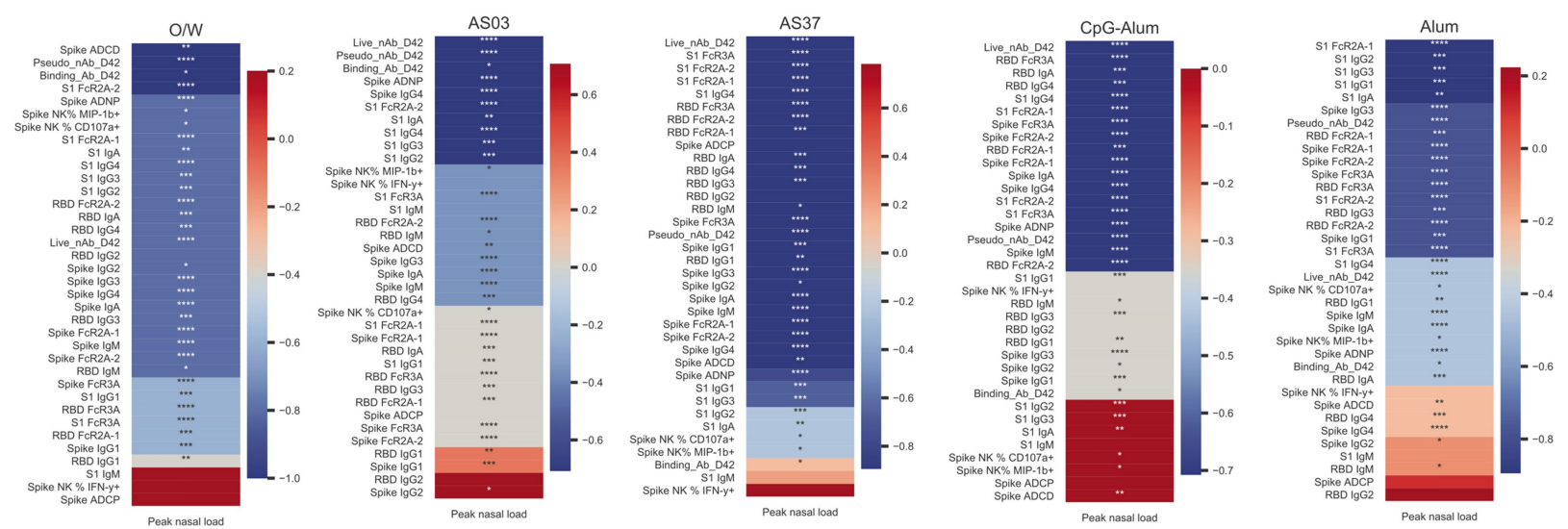

Extended Data Fig. 7 | Functional antibody profiling by systems serology. a-c, SARS-CoV-2 spike-specific binding $\operatorname{IgM}(\mathbf{a}), \operatorname{IgG1}(\mathbf{b})$ and $\operatorname{IgA}(\mathbf{c})$ responses in sera collected at days $-7,21$ and 42 . d, e, FcR-binding antibody responses FcR3A (d) and antibody-dependent neutrophil phagocytosis (ADNP) (e) measured in serum collected at days $-7,21$ and 42 .f, PLSDA analysis of all antibody features measured using systems serology. $g$, The top 3 antibody features discriminating protected vs. infected animals on day 42 in the PLSDA analysis. $\mathbf{h}$, Heat map showing Spearman's correlation between peak nasal viral load (left) or pharyngeal vial load (right) and antibody responses (day 42) indicated on the $y$ axis. $\mathbf{i}$, Heat map showing Spearman's correlation between peak nasal viral load and antibody responses within each group. In a-e, the boxes show median, 25 th and 75 th percentiles and the whiskers show the range. Asterisks represent statistically significant difference between two groups determined by two-sided Mann-Whitney rank-sum test. In $\mathbf{h}, \mathbf{i}$, the $P$ values were calculated for Spearman's correlation. ${ }^{*} P<0.05,{ }^{* *} P<0.01,{ }^{* * *} P<0.001$ and $^{* * * *} P<0.0001$. 
a

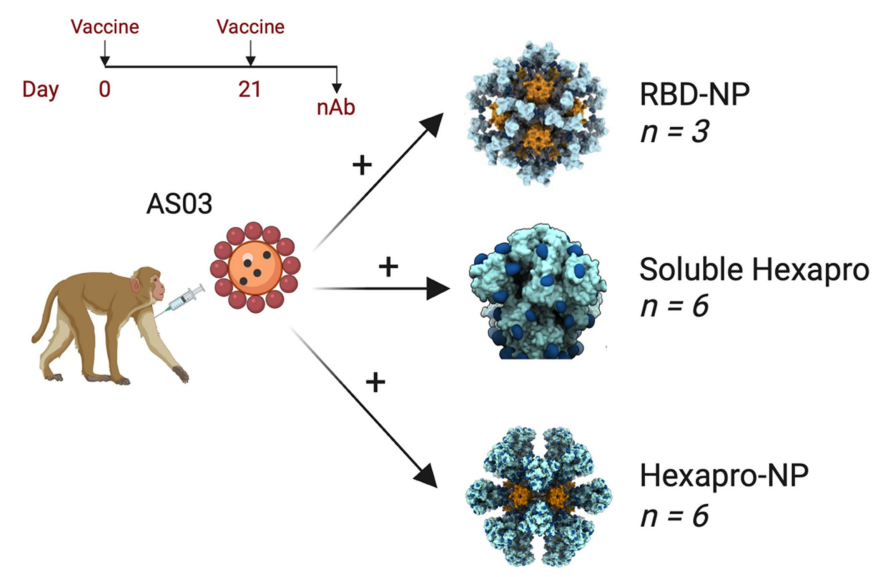

\section{b Pseudovirus nAb response}

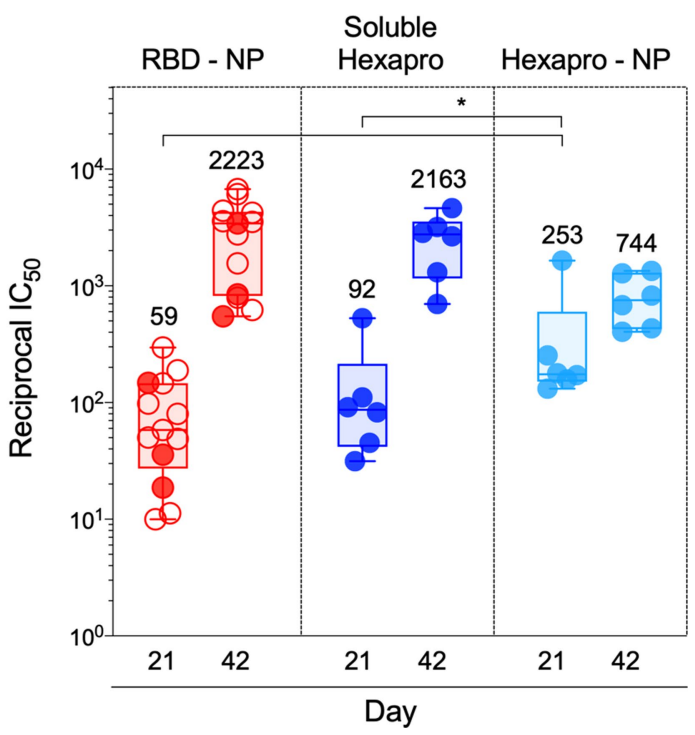

75th percentiles and the whiskers show the range. The numbers indicate GMT. Asterisks represent statistically significant differences between two groups analysed by two-sided Mann-Whitney rank-sum test $\left({ }^{*} P<0.05\right)$.
Extended Data Fig. 8 | RBD-NP or HexaPro immunization with ASO3 elicits comparable nAb responses. $\mathbf{a}$, A schematic of the study design. $\mathbf{b}$, Pseudovirus $\mathrm{nAb}$ responses in serum on day 21 and 42 . The box plots show median, 25 th and

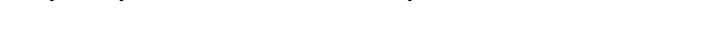


Article

\section{Extended Data Table 1 | List of animals used in the study}

\begin{tabular}{|c|c|c|c|c|}
\hline Animal ID & Antigen & Adjuvant & Age at enrollment & Weight at enrollment (kg) \\
\hline KM71 & No vaccine & No vaccine & 7 & 10.3 \\
\hline LA82 & No vaccine & No vaccine & 6 & 11.6 \\
\hline LP15 & No vaccine & No vaccine & 4 & 4.9 \\
\hline LD83 & No vaccine & No vaccine & 6 & 10 \\
\hline A11N092 & RBD-NP & $\mathrm{O} / \mathrm{W}$ & 9 & 10.3 \\
\hline KP93 & RBD-NP & $\mathrm{O} / \mathrm{W}$ & 7 & 10.1 \\
\hline LR25 & RBD-NP & $\mathrm{O} / \mathrm{W}$ & 4 & 5.1 \\
\hline LR27 & RBD-NP & $\mathrm{O} / \mathrm{W}$ & 4 & 5.8 \\
\hline KT40 & RBD-NP & AS03 & 7 & 10.3 \\
\hline LA51 & RBD-NP & AS03 & 6 & 9.5 \\
\hline LA71 & RBD-NP & $\mathrm{AS} 03$ & 6 & 10.1 \\
\hline LR32 & RBD-NP & AS03 & 4 & 5.8 \\
\hline LR36 & RBD-NP & AS03 & 4 & 6.2 \\
\hline LB08 & RBD-NP & AS37 & 6 & 10.9 \\
\hline LE83 & RBD-NP & AS37 & 6 & 11.4 \\
\hline LB31 & RBD-NP & AS37 & 6 & 9.5 \\
\hline LR59 & RBD-NP & AS37 & 4 & 6.2 \\
\hline LR71 & RBD-NP & AS37 & 4 & 6 \\
\hline LB62 & RBD-NP & CpG-Alum & 6 & 10.2 \\
\hline LC11 & RBD-NP & CpG-Alum & 6 & 9.7 \\
\hline LC38 & RBD-NP & CpG-Alum & 6 & 10.1 \\
\hline LR89 & RBD-NP & CpG-Alum & 4 & 5.1 \\
\hline LT80 & RBD-NP & CpG-Alum & 4 & 5.6 \\
\hline LD26 & RBD-NP & Alum & 6 & 9.1 \\
\hline LD35 & RBD-NP & Alum & 6 & 11 \\
\hline LD36 & RBD-NP & Alum & 6 & 9.6 \\
\hline LV86 & RBD-NP & Alum & 3 & 5 \\
\hline MA99 & RBD-NP & Alum & 3 & 4.6 \\
\hline LE42 & RBD-NP & AS03* & 6 & 11.7 \\
\hline LH90 & RBD-NP & AS03* & 6 & 11.2 \\
\hline LN06 & RBD-NP & AS03* & 4 & 5.3 \\
\hline LN69 & RBD-NP & AS03* & 4 & 4.6 \\
\hline LE62 & RBD-NP & AS03* & 6 & 8.5 \\
\hline LD44 & RBD-NP & AS03 & 6 & 10.8 \\
\hline LD54 & RBD-NP & AS03 & 6 & 9.5 \\
\hline MB72 & RBD-NP & AS03 & 3 & 5 \\
\hline MB84 & Soluble Hexapro & AS03 & 3 & 4.5 \\
\hline LB15 & Soluble Hexapro & AS03 & 6 & 11.8 \\
\hline LF37 & Soluble Hexapro & AS03 & 6 & 11.2 \\
\hline LI54 & Soluble Hexapro & AS03 & 5 & 9.6 \\
\hline LM34 & Soluble Hexapro & AS03 & 4 & 5.1 \\
\hline MC05 & Soluble Hexapro & AS03 & 3 & 4.2 \\
\hline LF69 & Hexapro NP & AS03 & 6 & 9.5 \\
\hline LE43 & Hexapro NP & AS03 & 6 & 11.4 \\
\hline $\begin{array}{l}\text { LI18 } \\
\text { LI18 }\end{array}$ & Hexapro NP & AS03 & 5 & 9.6 \\
\hline LI22 & Hexapro NP & AS03 & 5 & 9.0 \\
\hline LR71 & Hexapro NP & AS03 & 5 & 9.2 \\
\hline LI34 & Hexapro NP & AS03 & 3 & 4.9 \\
\hline
\end{tabular}

Animals marked with asterisks were not challenged. The grey shade indicates animals used in RBD-NP versus HexaPro comparison. 
Extended Data Table 2 | Cross-neutralization of SA B.1.351 in different adjuvant groups

\begin{tabular}{|c|c|c|c|c|c|c|}
\hline \multirow{2}{*}{ Group } & \multirow{2}{*}{ Animal } & \multicolumn{2}{|c|}{ Live-virus nAb $\left(\mathrm{IC}_{50}\right)$} & \multicolumn{2}{|c|}{ GMT } & \multirow{2}{*}{ Fold change } \\
\hline & & WT (Wuhan) & B.1.351 (SA) & WT (Wuhan) & B.1.351 (SA) & \\
\hline \multirow{4}{*}{$\mathrm{O} / \mathrm{W}$} & A11N092 & 131 & 10 & \multirow{4}{*}{300} & \multirow{4}{*}{53} & 13.1 \\
\hline & KP93 & 35 & 10 & & & 3.5 \\
\hline & LR25 & 1584 & 202 & & & 7.8 \\
\hline & LR27 & 1117 & 398 & & & 2.8 \\
\hline \multirow{10}{*}{$\begin{array}{c}\text { RBD- } \\
\text { NP/AS03 }\end{array}$} & KT40 & 999 & 238 & \multirow{10}{*}{2393} & \multirow{10}{*}{530} & 4.2 \\
\hline & LA51 & 1789 & 291 & & & 6.1 \\
\hline & LA71 & 1336 & 86 & & & 15.6 \\
\hline & LR32 & 2803 & 806 & & & 3.5 \\
\hline & LR36 & 4987 & 4334 & & & 1.1 \\
\hline & LE42 & 1302 & 209 & & & 6.2 \\
\hline & LH90 & 5320 & 1148 & & & 4.6 \\
\hline & LN06 & 2110 & 654 & & & 3.2 \\
\hline & LN69 & 1974 & 375 & & & 5.3 \\
\hline & LE62 & 6398 & 1429 & & & 4.5 \\
\hline \multirow{5}{*}{$\begin{array}{c}\text { RBD- } \\
\text { NP/AS37 }\end{array}$} & LB08 & 227 & 20 & \multirow{5}{*}{748} & \multirow{5}{*}{47} & 11.3 \\
\hline & LE83 & 273 & 10 & & & 28.4 \\
\hline & LB31 & 2541 & 117 & & & 21.8 \\
\hline & LR59 & 1517 & 93 & & & 16.2 \\
\hline & LR71 & 982 & 101 & & & 9.7 \\
\hline \multirow{5}{*}{$\begin{array}{l}\text { RBD- } \\
\text { NP/CpG- } \\
\text { Alum }\end{array}$} & LB62 & 889 & 11 & \multirow{5}{*}{1285} & \multirow{5}{*}{143} & 80.9 \\
\hline & LC11 & 1269 & 153 & & & 8.3 \\
\hline & LC38 & 635 & 75 & & & 8.5 \\
\hline & LR89 & 1496 & 681 & & & 2.2 \\
\hline & LT80 & 3268 & 698 & & & 4.7 \\
\hline \multirow{5}{*}{ Alum } & LD26 & 1919 & 412 & \multirow{5}{*}{1978} & \multirow{5}{*}{248} & 4.6 \\
\hline & LD35 & 1351 & 407 & & & 3.3 \\
\hline & LD36 & 4398 & 295 & & & 14.9 \\
\hline & LV86 & 1547 & 70 & & & 22 \\
\hline & MA99 & 1717 & 271 & & & 6.3 \\
\hline
\end{tabular}




\section{Reporting Summary}

Nature Research wishes to improve the reproducibility of the work that we publish. This form provides structure for consistency and transparency in reporting. For further information on Nature Research policies, see our Editorial Policies and the Editorial Policy Checklist.

\section{Statistics}

For all statistical analyses, confirm that the following items are present in the figure legend, table legend, main text, or Methods section.

n/a Confirmed

$\bigotimes$ The exact sample size $(n)$ for each experimental group/condition, given as a discrete number and unit of measurement

$\bigotimes$ A statement on whether measurements were taken from distinct samples or whether the same sample was measured repeatedly

The statistical test(s) used AND whether they are one- or two-sided

Only common tests should be described solely by name; describe more complex techniques in the Methods section.

Х $\square$ A description of all covariates tested

$\square$ \A description of any assumptions or corrections, such as tests of normality and adjustment for multiple comparisons

$\triangle$ A full description of the statistical parameters including central tendency (e.g. means) or other basic estimates (e.g. regression coefficient)

$\bigotimes$ AND variation (e.g. standard deviation) or associated estimates of uncertainty (e.g. confidence intervals)

For null hypothesis testing, the test statistic (e.g. $F, t, r$ ) with confidence intervals, effect sizes, degrees of freedom and $P$ value noted Give $P$ values as exact values whenever suitable.

Х $\square$ For Bayesian analysis, information on the choice of priors and Markov chain Monte Carlo settings

Х $\square$ For hierarchical and complex designs, identification of the appropriate level for tests and full reporting of outcomes

$\bigotimes$ Estimates of effect sizes (e.g. Cohen's $d$, Pearson's $r$ ), indicating how they were calculated

Our web collection on statistics for biologists contains articles on many of the points above.

\section{Software and code}

Policy information about availability of computer code

Data collection Flow cytometry data were collected using BD FACS Diva v.8.01 software associated with BD FACS Symphony. The number of foci in FRNT assay was acquired using CTL ImmunoSpot S6 Universal Analyzer. Luminescence was measured using a GloMax Navigator luminometer (Promega) for pseduovirus neutralization assay against B.1.1.7 variant. PET-CT images were acquired on a Mediso LFER 150 PET/CT (Mediso Medical Imaging Systems, Budapest, Hungary). Flow cytometry for systems serology was performed using an iQue (Intellicyt) and an S-LAB robot (PAA).

Data analysis FlowJo software v.10.0 (Treestar Inc); GraphPad Prism version 9.0.1; RStudio Version 1.2.1335; Adobe Illustrator 25.0. All statistical analysis are two-sided. FRNT foci were counted using Viridot, Version 3.12. PET-CT images were reconstructed using Nucline software 2.0. 3D images were created using OsiriX MD 12.0. Luminex analysis was performed on IntelliCyt ForeCyt ( 8 8.1) for Systems serology.

For manuscripts utilizing custom algorithms or software that are central to the research but not yet described in published literature, software must be made available to editors and reviewers. We strongly encourage code deposition in a community repository (e.g. GitHub). See the Nature Research guidelines for submitting code \& software for further information.

\section{Data}

Policy information about availability of data

All manuscripts must include a data availability statement. This statement should provide the following information, where applicable:

- Accession codes, unique identifiers, or web links for publicly available datasets

- A list of figures that have associated raw data

A description of any restrictions on data availability 


\section{Field-specific reporting}

Please select the one below that is the best fit for your research. If you are not sure, read the appropriate sections before making your selection.

$\bigotimes$ Life sciences $\quad \square$ Behavioural \& social sciences $\quad \square$ Ecological, evolutionary \& environmental sciences

For a reference copy of the document with all sections, see nature.com/documents/nr-reporting-summary-flat.pdf

\section{Life sciences study design}

All studies must disclose on these points even when the disclosure is negative.

Sample size No statistical test was used to determine the number of samples. Sample sizes were determined as appropriate to evaluate detection of large vaccine effects based on several studies done by us and others.

Data exclusions No data were excluded from any of the analysis.

Replication All the antibody assays were performed from each samples at least twice and the data were reproducible. All the other assays were performed once and all the biological replicates are presented.

Randomization Animals were randomly allocated to each group such that the median age and weight at the time of allocation was normalized across group. All the experiments were conducted with samples from each group represented in every experiment.

Blinding Investigators who performed neutralizing antibody, systems serology, challenge, viral load estimation, PET-CT were blinded during data collection and were unblinded after the analysis of the data. The rest of the experiments were conducted in an unblinded way since the investigators were involved in overall conduct of the study.

\section{Reporting for specific materials, systems and methods}

We require information from authors about some types of materials, experimental systems and methods used in many studies. Here, indicate whether each material, system or method listed is relevant to your study. If you are not sure if a list item applies to your research, read the appropriate section before selecting a response.

Materials \& experimental systems

\begin{tabular}{|c|c|}
\hline $\mathrm{n} / \mathrm{a}$ & Involved in the study \\
\hline & Х Antibodies \\
\hline & 区ukaryotic cell lines \\
\hline Х & Palaeontology and archaeology \\
\hline & \ Animals and other organisms \\
\hline & Х Human research participants \\
\hline$>$ & Clinical data \\
\hline$\searrow$ & Dual use research of concern \\
\hline
\end{tabular}

\begin{tabular}{l|l}
\multicolumn{2}{l}{ Methods } \\
\hline n/a & Involved in the study \\
\hline & $\square$ ChIP-seq \\
$\square$ & $\square$ Flow cytometry \\
$\square$ & $\square$ MRI-based neuroimaging
\end{tabular}

\section{Antibodies}

Antibodies used

Fluorochrome, Antibody, Vendor, Catalog\#, Clone, Lot\#, Usage, Reaction Volume per reaction (ul)

FITC, IL-2, Biolegend, 500304, MQ1-17H12, B268803, ICS, 2

PerCP-eF710, CXCR5, Invitrogen, 46-9185-42, MU5UBEE,2260300, Stimulation, 2.5

PE, IL-4, BioLegend , 500810, MP4-25D2, B267326, ICS, 1

PE-CF594, CD45RA, BD Biosciences, 565419, 5H9,0135941, Surface, 2

PE-Cy7, TNF-a, E-Bioscience, 25-7349-82, Mab11, E07679-1634, ICS, 0.3

BV421, CD40L, Biolegend, 310824, 24-31, B280078, ICS, 2

BV506, TCR-gd, Biolegend, 331220, B1.1, B288554, Surface, 2.5

BV605, CD4, Biolegend, 317438, OKT4,B289706, Surface, 1.5

BV650, CD3, BD Biosciences, 563916, SP34-2, B301629, Surface, 2.5

BV711, CCR7, Biolegend, 353228, G043H7, B284686, Surface, 2

BV785, CD127, Biolegend, 351330, A019D5, B283993, Surface, 2.5

APC, IL-21, BioLegend, 513008, 3A3-N2, B277959, ICS, 2.5

A700, IFN-g, Biolegend, 502520, 4S.B3, B302043, ICS, 1

APC-Cy7, CD25, Biolegend, 302614, BC96, B283801, Surface, 2

BUV395, CXCR3, BD Biosciences, 565223, 1C6/CXCR3, 9351552, Stimulation, 2.5

BUV563, CD8, BD Biosciences, 612914, RPA-T8, 0051261, Surface, 2

BUV737, CCR6, BD Biosciences, 612780, 11A9, 0265647, Surface, 2

BUV805, CD69, BD Biosciences, 748763, FN50, 0112825, Surface, 2

Anti-monkey IgG HRP, Alpha Diagnostics, 1:4,000, Lot XR9341-L 
APC-Cy7 mouse anti-human CD16, BD Bioscience, Cat\#557758, Lot\#0293144

Anti-CD66b pacblue, Biolegend, Cat\#305112, Lot\#B285068

CD56 PE-Cy7 mouse anti-human CD56, BD Biosciences, Cat\#557747, Lot\#0274120

CD107a PE-Cy5, BD Biosciences, Cat\#555802, Lot\#0149826

PE MIP-1b mouse anti-human, BD Biosciences, Cat\#550078, Lot\#0065243

PacBlue mouse anti-human CD3, BD Biosciences, Cat\#558117, Lot\#9332900

FITC Goat IgG anti-C3, MP Biomedical, Cat\#855385, Lot\#07829

Anti-rhesus IgG1, NHP reagent resource, Cat\#7H11, Lot\#013119EP

Anti-rhesus IgG2, NHP reagent resource, Cat\#3C10, Lot\#070815X

Anti-rhesus IgG3, NHP reagent resource, Cat\#2G11, Lot\#102611X

Anti-rhesus IgA, NHP reagent resource, Cat\#9B9, Lot\#021712AB

Anti-rhesus IgM, Life Diagnostics, Cat\#2C11-1-5, Lot\#C-I1515A

Goat anti-mouse IgG PE, Southern Biotech, Cat\#1030-09, Lot\#E2518-PE60

Validation

All antibodies used were evaluated by the manufacturers as provided in their websites. We did not perform any additional validation.

\section{Eukaryotic cell lines}

Policy information about cell lines

Cell line source(s)

HEK293T/17 is a female human embryonic kidney cell line (ATCC). The HEK-ACE2 adherent cell line was obtained through BEI Resources, NIAID, NIH: Human Embryonic Kidney Cells (HEK293T) Expressing Human Angiotensin-Converting Enzyme 2 HEK293T-hACE2 Cell Line, NR-52511. All adherent cells were cultured at $37^{\circ} \mathrm{C}$ with $8 \% \mathrm{CO} 2$ in flasks with DMEM $+10 \%$ FBS (Hyclone) $+1 \%$ penicillin-streptomycin. Vero E6 and THP-1 cells were purchased from ATCC.

Authentication

Cell lines were not authenticated.

Mycoplasma contamination

Cell lines were not tested for mycoplasma contamination nor authenticated.

Commonly misidentified lines

(See ICLAC register)

No commonly misidentified cell line was used in the study.

\section{Animals and other organisms}

Policy information about studies involving animals; ARRIVE guidelines recommended for reporting animal research

Laboratory animals All animals used in the study were male Rhesus Macaques (Macaca mulatta) of Indian origin, aged 3 - 10. A descriptive table containing age and weight of each animal is provided in Extended Data Table 1.

Wild animals

Study did not involve wild animals.

Field-collected samples

Study did not involve samples collected from the field.

Ethics oversight

The entire study (protocol 2020-8808-15) was reviewed and approved by the University of Louisiana at Lafayette Institutional Animal Care and Use Committee (IACUC). All animals were negative for SIV, simian T cell leukemia virus and simian retrovirus. For the challenge, the animals were transferred to the Regional Biosafety Level 3 facility at the Tulane National Primate Research Center, where the study was reviewed and approved by the Tulane University IACUC (Protocol 3918).

Note that full information on the approval of the study protocol must also be provided in the manuscript.

\section{Human research participants}

Policy information about studies involving human research participants

Population characteristics

Four human convalescent samples were assayed in parallel. They were obtained from individuals $37-67$ years of age.

Recruitment

The participants were recruited via print and online advertising from the Seattle metropolitan area as well as word of mouth. That means there is a bias towards people who live in Seattle and likely enriched for individuals associated with UW, though we did not track this.

Ethics oversight

The use of human convalescent sera samples was approved by the University of Washington Human Subjects Division Institutional Review Board (IRB00009810).

Note that full information on the approval of the study protocol must also be provided in the manuscript. 


\section{Plots}

Confirm that:

Х The axis labels state the marker and fluorochrome used (e.g. CD4-FITC).

Х The axis scales are clearly visible. Include numbers along axes only for bottom left plot of group (a 'group' is an analysis of identical markers).

$\bigotimes$ All plots are contour plots with outliers or pseudocolor plots.

$\bigotimes$ A numerical value for number of cells or percentage (with statistics) is provided.

\section{Methodology}

Sample preparation

Instrument

\section{Software}

Cell population abundance

Gating strategy

\Tick this box to confirm that a figure exemplifying the gating strategy is provided in the Supplementary Information.

\begin{abstract}
Live frozen PBMCs were revived, counted and resuspended at a density of 1 million live cells $/ \mathrm{ml}$ in complete RPMI (RPMI supplemented with $10 \%$ FBS and antibiotics). The cells were rested overnight at $37^{\circ} \mathrm{C}$ in $\mathrm{CO} 2$ incubator. Next morning, the cells were counted again, resuspended at a density of $15 \mathrm{million} / \mathrm{ml}$ in complete RPMI and $100 \mu \mathrm{l}$ of cell suspension containing 1.5 million cells was added to each well of a 96-well round-bottomed tissue culture plate. Each sample was treated with three conditions, no stimulation, a peptide pool spanning the RBD region of spike at a concentration of $1.2 \mu \mathrm{g} /$ $\mathrm{ml}$ of each peptide and a peptide pool spanning the I53-50A, and I53-50B components of the NP-scaffold $(1.2 \mu \mathrm{g} / \mathrm{ml}$ of each peptide) in the presence of $1 \mu \mathrm{g} / \mathrm{ml}$ of anti-CD28 (clone CD28.2, BD Biosciences) and anti-CD49d (clone 9F10, BD Biosciences) as well as anti-CXCR3 and anti-CXCR5 (clone and concentration details in supplementary table 2). The peptides were custom synthesized to $90 \%$ purity using GenScript, a commercial vendor. All samples contained $0.5 \% \mathrm{v} / \mathrm{v}$ DMSO in total volume of $200 \mu \mathrm{l}$ per well. The samples were incubated at $37^{\circ} \mathrm{C}$ in CO2 incubators for $2 \mathrm{~h}$ before addition of $10 \mu \mathrm{g} / \mathrm{ml}$ Brefeldin-A. The cells were incubated for an additional $4 \mathrm{~h}$. The cells were washed with PBS and stained with Zombie UV fixable viability dye (Biolegend). The cells were washed with PBS containing $5 \%$ FCS, before the addition of surface antibody cocktail (Supplementary table 1). The cells were stained for $20 \mathrm{~min}$ at $4^{\circ} \mathrm{C}$ in $100 \mu \mathrm{l}$ volume. Subsequently, the cells were washed, fixed and permeabilized with cytofix/cytoperm buffer (BD Biosciences) for 20 minutes. The permeabilized cells were stained with ICS antibodies for $20 \mathrm{~min}$ at room temperature in 1X-perm/wash buffer (BD Biosciences). Cells were then washed twice with perm/wash buffer and once with staining buffer before analysis using BD Symphony Flow Cytometer.
\end{abstract}

BD FACS Symphony

Data were acquired using BD FACS Diva v.8.01 and the data were analyzed using FlowJo software v.10.0.

No cell sorting procedure was used in the study.

Cells were selected based on FSC-A vs. SSC-A, singlets were selected using FSC-A vs. FSC-H. Live CD3 T cells were used for analysis of antigen-specific T cells. CD4 and CD8 T cells were selected as CD3+ CD4+ or CD3+ CD8+ after removal of gdT cells. 